\title{
SUR LES FONCTIONS ABÉLIENNES
}

PAR

\section{H. POINCARÉ}

à PAIS.

\section{S 1. Introduction.}

Je voudrais, sur la demande de M. Mittag-Leffler, présenter ici un exposé d'ensemble de mes travaux sur les fonctions abéliennes, en y ajoutant les quelques résultats nouveaux que j’ai pu obtenir dans ces derniers temps.

Une courbe $C$ de genre $p$ admet $p$ intégrales de première espèce

$$
u_{1}, u_{2}, \ldots, u_{p}
$$

et si cette courbe $C$ est coupée par une autre courbe algébrique variable $C^{\prime}$, le théorème d'Abel nous apprend que la somme des valeurs de l'intégrale $u_{k}$ aux divers points d'intersection est une constante.

Si la courbe $C^{\prime}$ est adjointe à $C$ et de degré suffisant (j'appelle $D$ ce degré) et que le nombre des points d'intersection de $C$ et de $C^{\prime}$ autres que les points doubles soit égal à $q$, on sait que $q-p$ de ces points peuvent être choisis à volonté.

Prenons alors $p$ points quelconques sur $C$; soient $M_{1}, M_{2}, \ldots, M_{p}$ ces points et considérons d'une part les $p$ sommes suivantes:

$$
v_{k}=u_{k \cdot 1}+u_{k \cdot 2}+\ldots+u_{k . p} \quad \quad(k=1,2, \ldots, p)
$$

où $u_{k . i}$ représente la valeur de l'intégrale $u_{k}$ au point $M_{i}$; et envisageons d'autre part un certain nombre de fonctions symétriques des coordonnées des $p$ points $M_{1}, M_{2}, \ldots, M_{p}$ et que j'appellerai les fonctions $\Phi$. 
Je pourrai choisir $p+\mathrm{I}$ fonctions $\Phi$ de telle façon que toutes les autres fonctions symétriques des coordonnées des points $M$ s'expriment rationnellement par le moyen de ces $p+\mathrm{I}$ fonctions, c'est à dire qu'à un système de valeurs de ces $p+\mathrm{I}$ fonctions ne puisse correspondre plus d'un système de $p$ points $M$. Ces $p+$ I fonctions seront d'ailleurs liées par une relation algébrique.

Ces $p+\mathrm{I}$ fonctions $\phi$ seront des fonctions analytiques des $v_{k}$; et ces fonctions seront holomorphes sauf peut-être en certains points singuliers. Je pourrai toujours trouver un système de points $M$ dans le voisinage desquels les fonctions $\Phi$ soient holomorphes. Je puis d'ailleurs supposer que ce système particulier de points correspond aux valeurs $v_{k}=0$; car les intégrales $u_{k}$ ne sont définies qu'à une constante près et je puis disposer de cette constante arbitraire de façon que $v_{k}$ s'annule pour ce système particulier de points. Dans ces conditions je puis dire que les fonctions $\Phi$ sont holomorphes pourvu que les modules des $v$ soient suffisamment petits.

Cela posé, choisissons sur $C$ un nombre $q-2 p$ de points fixes que j'appellerai

$$
A_{1}, A_{2}, \ldots, A_{q-2 p} .
$$

Soit $a_{k i}$ la valeur de l'intégrale $u_{k}$ au point $A_{i}$ et soit:

$$
\beta_{k}=\alpha_{k 1}+\alpha_{k 2}+\ldots+\alpha_{k \cdot q-2 p} .
$$

Par les $p$ points $M$ et par les $g-2 p$ points $A$ je puis faire passer une courbe adjointe $C^{\prime}$ de degré $D$ et une seule. Cette courbe coupe $C$ en tout en $q$ points en dehors des points doubles, elle passera donc encore par $p$ autres points que j'appelle

$$
M_{1}^{\prime}, M_{2}^{\prime}, \ldots, M_{p}^{\prime}
$$

J'appelle $u_{k i}^{\prime}$ la valeur de $u_{k}$ en $M_{i}^{\prime}$ et je pose:

$$
v_{k}^{\prime}=u_{k, 1}^{\prime}+u_{k .2}^{\prime}+\ldots+u_{k, p}^{\prime} .
$$

Soient $\Phi\left(v_{k}^{\prime}\right)$ les valeurs des fonctions $\Phi$ correspondant au système des $M^{\prime}$. A un système de points $M$ correspond un système de points $M^{\prime}$ et un seul puisque par les points $M$ et $A$ je ne puis faire passer qu'une seule courbe $C^{\prime}$. Il en résulte que les fonctions $\Phi\left(v_{k}^{\prime}\right)$ s'expriment rationnellement par le moyen des fonctions $\Phi\left(v_{k}\right)$. 
D'autre part le théorème d'Abel nous apprend que l'on a:

$$
v_{k}+\beta_{k}+v_{k}^{\prime}=r_{k}
$$

les $\gamma_{k}$ étant des constantes. Donc les $\Phi\left(v_{k}^{\prime}\right)=\Phi\left(\gamma_{k}-\beta_{k}-v_{k}\right)$ sont des fonctions rationnelles des $\Phi\left(v_{k}\right)$. Mais les points $A$ ont été choisis arbitrairement sur $C$; donc les constantes $\beta_{k}$ et par conséquent les $\gamma_{k}-\beta_{k}$ sont quelconques pourvu que le degré de $C^{\prime}$ ait été pris assez grand pour que $q \geqq 3 p$. Donc quelles que soient les constantes $\lambda_{k}$, les $\Phi\left(\lambda_{k}-v_{k}\right)$ sont des fonctions rationnelles des $\Phi\left(v_{k}\right)$.

Donc quelles que soient les constantes $\lambda$ et $\mu$, les

$$
\Phi\left(\lambda_{k}+v_{k}\right)=\Phi\left[\left(\lambda_{k}+\mu_{k}\right)-\left(\mu_{k}-v_{k}\right)\right]
$$

sont des fonctions rationnelles des $\Phi\left(\mu_{k}-v_{k}\right)$ qui sont eux-mêmes des fonctions rationnelles des $\Phi\left(v_{k}\right)$. Les $\Phi\left(\lambda_{k}+v_{k}\right)$ sont donc des fonctions rationnelles des $\Phi\left(v_{k}\right)$; en permutant le rôle des quantités $\lambda_{k}$ et $v_{k}$, on voit que ce sont aussi des fonctions rationnelles des $\Phi\left(\lambda_{k}\right)$. Les $\Phi\left(\lambda_{k}+v_{k}\right)$ sont donc des fonctions rationnelles des $\Phi\left(v_{k}\right)$ et des $\Phi\left(\lambda_{k}\right)$. Si nous faisons $\lambda_{k}=v_{k}$, nous voyons que les $\Phi\left(2 v_{k}\right)$ sont des fonctions rationnelles des $\Phi\left(v_{k}\right)$.

Or j'ai dit que les $\Phi\left(v_{k}\right)$ sont holomorphes si le module de $v_{k}$ est suffisamment petit, plus petit que $\rho$ par exemple. Alors les $\Phi\left(2 v_{k}\right)$ qui sont rationnels par rapport aux $\Phi\left(v_{k}\right)$ seront holomorphes si $|v|<\rho$; donc les $\Phi\left(v_{k}\right)$ sont méromorphes si $|v|<2 \rho$. Mais alors les $\Phi\left(2 v_{k}\right)$ étant rationnels par rapport aux $\Phi\left(v_{k}\right)$ seront méromorphes pourvu que $|v|<2 \rho$. Donc les $\Phi\left(v_{k}\right)$ seront méromorphes pourvu que $|v|<4 \rho$; et ainsi de suite.

En résumé les $\Phi$ sont des fonctions uniformes et méromorphes des $v$ pour toutes les valeurs de ces variables.

Quand l'un des points $M$ décrit un cycle sur la surface de Rremann correspondant à la courbe $C$, les fonctions $\Phi$ reviennent à leurs valeurs primitives, et les intégrales $u_{k}$ et par conséquent les $v_{k}$ augmentent d'une période. Il en résulte que les fonctions $\Phi$ sont périodiques; ce sont des fonctions à $p$ variables et à $2 p$ périodes.

C'est ainsi qu'on a été conduit aux fonctions abéliennes, et Riemans a montré comment on peut les exprimer par le moyen des fonctions $\boldsymbol{\theta}$.

Mais une courbe de genre $p$, dépend de $3 p-3$ paramètre, tandis qu'une fonction $\theta$ de $p$ variables dépend de $\frac{p(p+1)}{2}$ paramètres. Ce second 
nombre est plus grand que le premier sauf pour $p=2$ et pour $p=3$. $\Pi$ y a donc des fonctions $\theta$ de $p$ variables qui ne peuvent pas être engendrées par une courbe de genre $p$ de la façon que je viens de dire; aussi appellerai-je fonctions $\theta$ spéciales et fonctions abéliennes spéciales celles qui sont susceptibles de ce mode de génération.

Une des premières questions à résoudre est donc de reconnaître les relations des fonctions abéliennes spéciales avec les fonctions abéliennes générales et les caractères qui les différencient les unes des autres.

Mais une autre question se pose. Riemans a démontré qu'il y a une certaine relation entre les périodes d'une fonction abélienne spéciale. $\mathrm{Si}$ cette relation a lieu, on peut former la fonction $\theta$; si elle n'a pas lieu, la fonction $\theta$ n'existe pas. Nous venons de voir qu'il y a des fonctions $\theta$ qui ne sont pas spéciales, et par conséquent des fonctions $2 p$ fois périodiques qui ne sont pas spéciales et dont les périodes sont cependant liées par la relation de Riemann.

Mais ne peut-il pas exister aussi des fonctions $2 p$ fois périodiques dont les périodes ne sont pas liées par une semblable relation et qui ne peuvent pas s'exprimer par les fonctions $\theta$ ? La réponse doit être négative. 'Toute fonction à $p$ variables et $2 p$ périodes peut s'exprimer par les fonctions $\boldsymbol{\theta}$.

C'est là un théorème que j'appellerai le théorème $B$ et sur lequel je reviendrai dans la suite.

Mais je dois d'abord parler d'un autre théorème que j'appellerai le théorème $A$ et d'après lequel entre $p+1$ fonctions à $p$ variables et à $2 p$ périodes il $\mathrm{y}$ a toujours une relation algébrique.

\section{§ 2. Démonstration du théorème A.}

Théorème A. Si l'on a $p+$ I fonctions de $p$ variables, méromorphes pour toutes les valeurs de ces $p$ variables et admettant $2 p$ périodes distinctes, ces fonctions sont liées par une relation algébrique.

Dans la plupart des démonstrations du théorème $B$, on s'appuie sur le théorème $A$ et on a déjà proposé plusieurs démonstrations de ce théorème $A$. On pourrait d'ailleurs s'en passer puisque je donnerai plus loin une 
démonstration du théorème $B$, indépendante du théorème $A$ et que d'ailleurs rien n'est plus facile que de déduire le théorème $A$ du théorème $B$.

Je ne crois pourtant pas inutile de développer ici une démonstration nouvelle du théorème $A$ que je n'avais fait qu'esquisser dans les Comptes Rendus en 1897.

Soient $F_{1}, F_{2}, \ldots, F_{p}^{\prime}$, des fonctions des $p$ variables $v$, méromorphes pour toutes les valeurs finies de ces variables et admettant $2 p$ périodes. Je considère les zéros communs à ces $p$ fonctions qui sont à l'intérieur du prismatoïde des périodes.

\section{1 ère proposition.}

Je dis d'abord que ces zéros communs, ou bien sont en nombre fini, ou bien forment un continuum.

Soit en effet

$$
v_{1}=\alpha_{1}, \quad v_{2}=\alpha_{2}, \ldots, \quad v_{p}=\alpha_{p}
$$

un des zéros communs des fonctions $F$, c'est à dire un système de valeurs des $v$ pour lequel on a:

$$
F_{1}=F_{2}=\ldots=F_{p}=0 \text {. }
$$

D'après notre hypothèse, les fonctions $F$ sout méromorphes pour toutes les valeurs des $v$; cela signifie que si l'on considère les parties réelles et imaginaires des $v$ comme les coordonnées d'un point dans l'espace à $2 p$ dimensions l'on peut construire dans cet espace une série de domaines $D_{1}, D_{2}, \ldots$ qui seront par exemple des hypersphères et cela de telle façon:

$I^{\circ}$ que tout point de l'espace à $2 p$ dimensions appartienne au moins à l'un de ces domaines.

$2^{\circ}$ que si $R$ est une région finie quelconque de cet espace, par exemple le prismatoïde des périodes, il n'y a qu'un nombre fini de domaines $D$ qui soient en totalité ou en partie contenus dans la région $R$.

$3^{\circ}$ qu'à l'intérieur de chaque domaine $D$, on ait:

$$
F_{1}=\frac{P_{1}}{Q_{1}}, \quad F_{2}=\frac{P_{2}}{Q_{2}}, \ldots, \quad F_{p}=\frac{P_{p}}{Q_{p}},
$$

les fonctions $P_{1}, P_{2}, \ldots, P_{p} ; Q_{1}, Q_{2}, \ldots, Q_{p}$; étant holomorphes dans tout le domaine $D$. 
Si maintenant deux des domaines $D$ et $D_{1}$ ont une partie commune (ce qui arrivera forcément, car, puisque nous voulons que tout point de l'espace soit intérieur au moins à l'un de nos domaines, il doit exister des régions de l'espace communes à plusieurs domaines) nous avons dans le domaine $D$ :

$$
F_{1}=\frac{P_{1}}{Q_{1}}
$$

dans le domaine $D_{1}$ :

$$
F_{1}=\frac{P_{1}^{\prime}}{Q_{1}^{\prime}}
$$

et par conséquent dans la partie commune

$$
F_{1}=\frac{P_{1}}{Q_{1}}=\frac{P_{1}^{\prime}}{Q_{1}^{\prime}}
$$

Il ne s'ensuit pas que dans cette partie commune $P_{1}$ est égal à $P_{1}^{\prime}$ et $Q_{1}$ à $Q_{1}^{\prime}$. J'ai démontré il est vrai dans le tome 2 des Acta mathematica un théorème d'après lequel toute fonction méromorphe dans tout l'espace peut toujours être considérée comme le quotient de deux fonctions holomorphes dans tout l'espace. Depuis $M$. Cousin a donné dans sa thèse une démonstration de ce même thèorème.

Il en résulterait alors que les deux fonctions holomorphes $P_{1}$ et $Q_{1}$ pourraient être supposées les mêmes dans tous les domaines. Mais je voudrais éviter pour le moment de m'appuyer sur ce théorème.

\section{Démonstration de la $\mathrm{I}^{\mathrm{i} \mathrm{e} e}$ proposition.}

Il s'agit de démontrer qu'à l'intérieur du domaine $D$, les $p$ fonctions holomorphes

$$
P_{1}, P_{2}, \ldots P_{p}
$$

ne peuvent avoir un nombre infini de zéros, à moins que l'ensemble de ces zéros ne forme un continuum analytique.

Si en effet ces fonctions possédaient un nombre infini de zéros communs, à l'intérieur de $D$, il devrait y avoir dans $D$ un point:

$$
v_{1}=a_{1}, \quad v_{2}=\alpha_{2}, \ldots, v_{p}=a_{p}
$$


dans le voisinage duquel il y aurait une infinité de zéros communs à nos $p$ fonctions. Cela posé; supposons que nos $p$ équations

$$
P_{1}=P_{2}=\ldots=P_{p}=\text { o }
$$

ne se réduisent pas toutes à des identités; que par exemple $P_{1}$ ne soit pas identiquement nul. Je pourrai supposer que $P_{1}=0$ ne se réduit pas à une identité quand on fait

$$
v_{2}=\alpha_{2}, \quad v_{3}=\alpha_{3}, \ldots, \quad v_{p}=\alpha_{p} .
$$

$\mathrm{Si}$ en effet cela avait lieu on pourrait tourner la difficulté par un simple changement linéaire de variables. Comme $P_{1}$ n'est pas identiquement nul, il y a toujours un point

$$
v_{1}=\beta_{1}, \quad v_{2}=\beta_{2}, \ldots, \quad v_{p}=\beta_{p}
$$

pour lequel $P_{1}$ n'est pas nul. Soient alors $v_{1}^{\prime}, v_{2}^{\prime}, \ldots, v_{p}, p$ combinaisons linéaires des $v ; \alpha_{1}^{\prime}, \alpha_{2}^{\prime}, \ldots, \alpha_{p}^{\prime}$ les combinaisons linéaires correspondantes des $a . \beta_{1}^{\prime}, \beta_{2}^{\prime}, \ldots, \beta_{p}^{\prime}$ les combinaisons linéaires correspondantes des $\beta$. Je pourrai choisir les coefficients de ces combinaisons linéaires de telle sorte que:

$$
\beta_{2}^{\prime}=\alpha_{2}^{\prime}, \quad \beta_{3}^{\prime}=\alpha_{3}^{\prime}, \ldots, \quad \beta_{p}^{\prime}=\alpha_{p}^{\prime} .
$$

Je prendrai alors les $v^{\prime}$ comme nouvelles variables et je verrai que l'équation $P_{1}=\mathrm{o}$ ne se réduit pas à une identité pour

$$
v_{2}^{\prime}=a_{2}^{\prime}, \quad v_{s}^{\prime}=a_{s}^{\prime}, \ldots, \quad v_{p}^{\prime}=a_{p}^{\prime} .
$$

Supposons donc que $P_{1}$ ne s'annule pas identiquement pour $v_{2}=a_{2}$, $v_{3}=\alpha_{3}, \ldots, v_{p}=\alpha_{p}$. Alors en vertu des lemmes que j'ai démontrés au début de ma Thèse Inaugurale, l'équation $P_{1}=0$ definira $v_{1}-\alpha_{1}$ en fonction algébroïde de $v_{2}-a_{2}, v_{3}-a_{3}, \ldots, v_{p}-a_{p}$. Donc en vertu des mêmes lemmes $P_{2}, P_{3}, \ldots, P_{p}$ seront aussi des fonctions algébroïdes de $v_{2}-\alpha_{2}, \ldots, v_{p}-\alpha_{p}$ et les équations

$$
P_{\mathrm{g}}=P_{\mathrm{s}}=\ldots=P_{\mathrm{p}}=0
$$

pourront être remplacées par les suivantes

$$
P_{2}^{\prime}=P_{\mathrm{s}}^{\prime}=\ldots=P_{p}^{\prime}=\text { o }
$$

Acta mallomatiea. 26. Imprimé le 11 avril 1902. 
où les $P^{\prime}$ sont des fonctions holomorphes de

$$
v_{2}-\alpha_{2}, v_{3}-\alpha_{3}, \ldots, v_{p}-\alpha_{p} .
$$

Les équations (2) sont de même forme que les équations (I), seulement le nombre des équations comme celui des variables est diminué d'une unité.

$\mathrm{Si}$ ces équations (2) se réduisent à des identités, l'équation $P_{1}=0$ suffit pour définir les zéros communs qui forment un continuum analytique à $p$ - I dimensions.

Si les équations (2) ne se réduisent pas à des identités, on opérera sur elles comme sur les équations ( I).

On ne sera arrêté que quand on arrivera à un système d'équations se réduisant à des identités, auquel cas l'ensemble des zéros formera un continuum analytique; ou quand le système se réduira à une équation à une inconnue. Mais pour une équation à une inconnue dont le premier membre est holomorphe et qui ne se réduit pas à une identité, on sait qu'il ne peut pas $y$ avoir de point dans le voisinage duquel il $y$ ait une infinité de zéros.

La proposition est donc démontrée.

Remarque. Pour appliquer ce qui précède aux fonctions méromorphes $F_{1}, F_{2}, \ldots, F_{p}$, il faut préciser ce que nous devons entendre par un zéro de $F_{1}$. D'abord $F_{1}$ ne pourra pas s'annuler sans que $P_{1}$ s'annule. Tous les zéros de $F_{1}$ appartiennent donc à $P_{1}$; mais il peut arriver qu'un zéro de $P_{1}$ n'appartienne pas à $F_{1}$. Soit en effet $v_{i}=a_{i}$ un zéro de $P_{1}$; supposons que $P_{1}$ et $Q_{1}$ soient divisibles par une même fonction $H$, holomorphe dans le voisinage de $v_{i}=\alpha_{i}$ et s'annulant pour $v_{i}=\alpha_{i}$, de telle sorte que

$$
P_{1}=H P_{1}^{\prime}, \quad Q_{1}=H Q_{1}^{\prime},
$$

$P_{1}^{\prime}$ et $Q_{1}^{\prime}$ étant holomorphes pour $v_{i}=\alpha_{i}$. Il pourrait se faire que $P_{1}$ et $H$ s'annulant pour $v_{i}=\alpha_{i}, P_{1}^{\prime}$ ne s'annulât pas pour $v_{i}=a_{i}$. Dans ce cas $v_{i}=\alpha_{i}$ serait un zéro de $P_{1}$, mais ne serait pas un zéro de

$$
F_{1}=\frac{P_{1}}{Q_{1}}=\frac{P_{1}^{\prime}}{Q_{1}^{\prime}}
$$

Reprenons alors la démonstration précédente et cherchons comme tont à l'heure les zéros communs à $F_{1}, F_{2}, \ldots, F_{p}$ et voisins de $v_{i}=\alpha_{i}$. Je suppose que $P_{1}$ et $Q_{1}$ soient divisible par $H_{1} ; P_{2}$ et $Q_{3}$ par $H_{2} ; \ldots ; P_{p}$ 
et $Q_{p}$ par $H_{p} ;$ les fonctions $H_{1}, H_{2}, \ldots, H_{p}$ étant holomorphes, dans le voisinage de $v_{i}=\alpha_{i}$ et nulles pour $v_{i}=a_{i}$. Nous remplacerons alors les équations (I) par les équations

$$
\frac{P_{1}}{H_{1}}=\frac{P_{2}}{H_{2}}=\ldots=\frac{P_{p}}{H_{p}}=\text { o. }
$$

Ce sont les équations ( $I^{\prime}$ ) et non plus les équations (I) qui nous donneront les zéros communs à $F_{1}, F_{2}, \ldots, F_{p}$; mais les équations (I') étant de même forme que les équations (I) il n'y a rien à changer au raisonnement.

Supposons maintenant que l'on envisage la partie commune aux deux domaines $D$ et $D_{1}$ et que dans cette partie commune on ait:

$$
F_{k}=\frac{P_{k}}{Q_{k}}=\frac{P_{k}^{\prime}}{Q_{k}^{\prime}}
$$

que $P_{k}$ et $Q_{k}$ soient divisibles par $H_{k} ; P_{k}^{\prime}$ et $Q_{k}^{\prime}$ par $H_{k}^{\prime}$; les fonctions $H_{k}$ et $H_{k}^{\prime}$ étant holomorphes pour $v_{i}=\alpha_{i}$ et s'annulant pour $v_{i}=\alpha_{i}$. Supposons enfin que $\frac{P_{k}}{H_{k}}$ et $\frac{Q_{k}}{H_{k}}$ ne soient divisibles à la fois par aucune fonction holomorphe s'annulant pour $v_{i}=\alpha_{i}$, et qu'il en soit de même pour $\frac{P_{k}^{\prime}}{\boldsymbol{H}_{k}^{\prime}}$ et $\frac{Q_{k}^{\prime}}{\boldsymbol{H}_{k}^{\prime}}$. Alors le système des équations ( $\mathrm{I}^{\prime}$ ) est équivalent au système des équations

$$
\frac{P_{1}^{\prime}}{H_{1}^{\prime}}=\frac{P_{2}^{\prime}}{H_{2}^{\prime}}=\ldots=\frac{P_{p}^{\prime}}{H_{k}^{\prime}}=\text { o. }
$$

\section{$2^{e}$ proposition.}

Si les fonctions $P_{1}, P_{2}, \ldots, P_{p}$ qui sont holomorphes dans le domaine $D$ dépendent d'un ou plusieurs paramètres $\lambda$ que l'on peut faire varier d'une manière continue; si on considère ceux des zéros communs aux $p$ fonctions $P$ qui sont à l'intérieur d'un domaine $\Delta$ intérieur lui-même à $D$; si quand on fait varier les paramètres $\lambda$ entre certaines limites, le nombre des zéros communs reste fini; ce nombre ne peut varier que si un des zéros se déplaçant d'une manière continue sort du domaine $\Delta$ ou $\mathrm{y}$ entre, en traversant la frontière de ce domaine.

Nous savons en effet que Cauchy a exprimé le nombre des zéros de $f(z)$ situés à l'intérieur d'un contour par l'intégrale définie:

$$
\frac{1}{2 i \pi} \int \frac{f^{\prime} d s}{f}
$$


prise le long de ce contour, et que KroneCKer, généralisant le théorème de Cauchy, a exprimé le nombre des zéros communs à $P_{1}, P_{2}, \ldots, P_{p}$ à l'intérieur de $\Delta$ par une intégrale multiple prise le long de la frontière de $\Delta$.

La fonction sous le signe $\int$ reste d'ailleurs finie et continue sauf pour les zéros communs aux fonctions $P$, de sorte que si aucun de ces zéros ne se trouve sur la frontière de $\Delta$, l'intégrale de Kronecker reste finie et est une fonction continue des $\lambda$.

Si le nombre des zéros communs est infini, il y en a en général sur la frontière de $\Delta$ et l'intégrale est dans ce cas dépourvue de sens. Mais si ce nombre est fini, il n'y en aura pas en général sur la frontière de $\Delta$; s'il $y$ en avait, il suffirait pour tourner la difficulté de déformer infiniment peu le domaine $\Delta$.

L'intégrale de Kronecker est une fonction continue des $\lambda$, tant qu'il n'y a pas de zéros communs sur la frontière de $\Delta$, et comme sa valeur est toujours un nombre entier elle doit être constante. Le nombre de ces zéros communs ne peut donc varier que quand l'un d'eux vient sur la frontière de $\Delta$.

C. Q. F. D.

Remarque. Le théorème s'applique d'ailleurs immédiatement aux fonctions méromorphes $F_{1}, F_{2}, \ldots, F_{p}$, puisque les zéros communs de ces fonctions sont ceux des fonctions holomorphes que nous appelions plus haut $\frac{P_{1}}{H_{1}}, \frac{P_{2}}{H_{q}}, \ldots, \frac{P_{p}}{H_{p}}$.

Considérant maintenant un domaine $\Delta$ formé de plusieurs domaines partiels $\Delta_{1}, \Delta_{2}, \ldots, \Delta_{p}$; et supposons que dans chacune de ces domaines partiels la fonction $F_{i}$ puisse se mettre sous la forme $\frac{P_{i}}{Q_{i}}$, le théorème s'applique au domaine total $\Delta$.

En effet le nombre des zéros intérieurs à l'un des domaines partiels $\Delta_{q}$ ne peut varier que si un zéro franchit la frontière de ce domaine; alors de deux choses l'une, ou bien la partie de la frontière de $\Delta_{q}$ franchie par le zéro appartient à la frontière du domaine total $\Delta$, et alors notre zéro a franchi la frontière de $\Delta$; ou bien cette partie de la frontière de $\Delta_{q}$ sépare $\Delta_{q}$ d'un autre domaine partiel $\Delta_{p}$, et alors le nombre des zéros intérieurs à $\Delta_{q}$ aura diminué d'une unité; mais en même temps celui des 
zéros intérieurs à $\Delta_{p}$ aura augmenté d'une unité; le nombre total des zéros intérieurs à $\Delta$ n'aura pas changé.

En résumé le nombre des zéros intérieurs à $\Delta$ ne peut varier que si un zéro franchit la frontière de $\Delta$.

$$
\text { C. Q. F. D. }
$$

Corollaire. Ce que nous avons dit jusqu'ici s'applique ì toutes les fonctions méromorphes; nous allons voir maintenant ce qui est particulier aux fonctions abéliennes.

Je prendrai pour le domaine $\Delta$ le prismatoïde des périodes.

Je vois que le nombre des zéros communs aux $p$ fonctions $F$ intérieurs à ce prismatoïde est fini, à moins que leur ensemble ne forme un continuum analytique; et que, quand on fait varier les $\lambda$, ce nombre, s'il reste fini, ne peut varier que quand un zéro franchit la frontière du prismatoïde. Il augmente d'une unité toutes les fois qu'un zéro entre dans le prismatoïde, et il diminue d'une unité toutes les fois qu'un zéro en sort.

Mais, à cause de la périodicité supposée de nos fonctions, un zéro ne peut entrer dans le prismatoïde sans qu'un autre en sorte; le nombre de nos zéros, s'il reste 'fini, demeure donc constant.

\section{$3^{e}$ proposition.}

Soient $F_{1}^{\prime}, F_{2}^{\prime}, \ldots, F_{p+1}, p+\mathrm{I}$ fonctions abéliennes à $p$ variables. Soient

$$
\Phi_{1}, \Phi_{2}, \ldots, \Phi_{p}
$$

$p$ combinaisons linéaires de ces $p+1$ fonctions de telle sorte que:

$$
\Phi_{q}=\alpha_{q, 1} F_{1}+\alpha_{q, 2} F_{2}+\ldots+\alpha_{q, p+1} F_{p+1}+\alpha_{q, p+2}
$$

le nombre des zéros communs aux $p$ fonctions $\Phi$, tant qu'il reste fini, est indépendant des coefficients $\alpha$.

C'est là une conséquence immédiate des propositions précédentes.

Le résultat peut s'énoncer dans le langage géométrique.

Considérons $F_{1}, F_{2}, \ldots, F_{p+1}$ comme les coordonnées d'un point dans l'espace à $p+1$ dimensions; comme ces fonctions dépendent seulement de $p$ variables, ce point sera sur une certaine variété à $p$ dimensions que 
j'appellerai $V$; notre but est précisément de démontrer que cette variété est algébrique et nous voyons déjà d'après l'ensemble des résultats obtenus, que le nombre des points d'intersections de cette variété avec une droite quelconque est fini, à moins que la droite ne soit tout entière sur la variété et que ce nombre est constant pour toutes les droites qui ne sont pas tout entières sur la variété. Soit $q$ ce nombre.

Considérons maintenant, au lieu d'une droite, une courbe algébrique quelconque $C$ d'ordre $n$ : je dis que le nombre des points d'intersection de cette courbe avec $V$ sera $n q$ à moins que la courbe ne soit tout entière sur la variété ou qu'elle se décompose en plusieurs composantes, une de ces composantes étant tout entière sur la variété.

Il me suffira de démontrer cela pour les courbes planes, c'est à dire pour les courbes situées sur une variété plane à 2 dimensions. L'équation générale d'une pareille courbe est:

$$
\boldsymbol{\theta}=\mathrm{o}, \quad \Phi_{1}=\Phi_{2}=\ldots=\Phi_{p-1}=0 ;
$$

$\boldsymbol{\theta}$ étant un polynôme entier d'ordre $n$ et les $\boldsymbol{\phi}$ des polynômes du ${ }^{\prime \prime}$ ordre par rapport aux $F$. Le nombre des points d'intersection, tant qu'il reste fini, est indépendant des coefficients du polynôme $\theta$; or quand ce polynôme $\boldsymbol{\theta}$ se décompose en $n$ facteurs linéaires, ce nombre est évidemment $n q$. Et d'un autre côté ce nombre ne peut devenir infini que si l'ensemble des points d'intersection forme un continuum analytique, c'est à dire qu'il doit être formé de tous les points de l'une au moins des composantes de notre courbe $C$.

La même démonstration s'appliquerait sans changement à une courbe qui serait une »intersection complète", c'est à dire dont l'équation s'écrirait:

$$
\theta_{1}=\theta_{2}=\ldots=\theta_{p}=0
$$

les $\theta$ étant des polynomes de degré quelconque. Pour étendre enfin le résultat à une courbe $C^{\prime}$ qui ne serait pas une intersection complète, il suffit d'observer qu'on peut toujours trouver une intersection complète qui se compose de $C$ et d'un certain nombre de droites.

\section{$4^{e}$ proposition.}

La variété $V$ est algébrique. 
Soit en effet $\theta$ un polynôme entier quelconque d'ordre $n$ en $\boldsymbol{F}_{1}, \boldsymbol{F}_{2}, \ldots$, $F_{p+1}$. Il contient

$$
\frac{(n+p+\mathrm{r})}{\underline{n(p+\mathrm{r})}}
$$

coefficients arbitraires.

Soit

$$
v_{1}=\alpha_{1}, \quad v_{2}=\alpha_{2}, \ldots, \quad v_{p}=\alpha_{p}
$$

un système quelconque de valeurs des $v$; nous allons chercher à annuler la fonction $\theta$ et ses dérivées d'ordre $\mathrm{I}, 2, \ldots, q$ par rapport aux $p$ variables $v$ pour le point $v_{i}=\alpha_{i}$. Ces dérivées étant au nombre de

$$
\frac{\mid(n q+p)}{|\underline{n q}| \underline{p}}
$$

cela sera possible pourvu que

$$
\frac{\mid(n+p+I)}{\mid \underline{n \mid \underline{p+I})}}>\frac{(n q+p)}{\underline{n q} \underline{p}} .
$$

Or on peut toujours prendre $n$ assez grand pour satisfaire à cette inégalité, puisque le $\mathrm{I}^{\text {er }}$ membre est un polynôme d'ordre $p+\mathrm{I}$ en $n$ et le second membre un polynôme d'ordre $p$.

La variété $\theta=0$, aura alors avec la variété $V$ un contact d'ordre $n q$ au point $v_{i}=\alpha_{i}$. Un plan quelconque à 2 dimensions passant par le point $v_{i}=\alpha_{i}$ coupera la variété $\boldsymbol{\theta}=0$ suivant une courbe $C$ algébrique d'ordre $n$ qui aura avec $V$ un contact d'ordre $n q$. Cette courbe $C$ coupera donc $V$ en $n q+1$ points confondus avec le point $v_{i}=\alpha_{i}$.

Il en résulte qu'elle sera tout entière sur $V$ ou qu'elle se décomposera, l'une de ses composantes $C^{\prime}$ étant tout entière sur $C$.

L'ensemble des courbes $C^{\prime}$ engendrera une variété $V^{\prime}$ à $p$ dimensions. En effet, une droite quelconque $D$ de l'espace à $p+1$ dimensions rencontrera $V^{\prime}$; car par $D$ et par le point $v_{i}=a_{i}$ je puis faire passer un plan. Dans ce plan se trouvera d'après ce qui précède une des courbes $C^{\prime}$ et cette courbe $C^{\prime}$ rencontrera $D$; car une droite et une courbe algébrique situées dans un même plan se rencontrent toujours soit à distance finie, soit à l'infini. Donc $D$ rencontre $V^{\prime}$, puisque cette courbe $C^{\prime}$ est sur $V^{\prime}$. C. Q. F. D. 
Toutes les courbes $C^{\prime}$ étant sur la variété $\theta=0$, la variété $V^{\prime}$ sera tout entière sur la variété algébrique $\boldsymbol{\theta}=\mathrm{o}$. Done ou bien ces deux variétés à $p$ dimensions sont identiques, ou bien la variété algébrique $\boldsymbol{\theta}=\mathrm{o}$ se décompose et $V^{\prime}$ est l'une de ces composantes; $V^{\prime}$ est done algébrique.

Toutes les courbes $C^{\prime}$ étant sur $V$, la variété $V^{\prime}$ sera tout entière sur $V$ et comme la variété $V$ ne se décompose pas puisqu'à chaque système de valeurs des $v$ correspond un point de $V$ et un seul; ces deux variétés doivent être identiques.
Donc $V$ est algébrique.
C. Q. F. D.

Iue théorème $A$ est donc démontré.

Il pourrait $y$ avoir une difficulté si la variété $V$ avait moins de $p$ dimensions, c'est à dire s'il $\mathrm{y}$ avait une relation entre les $p$ fonctions $F_{1}$, $F_{2}, \ldots, F_{p} ;$ mais je dis quon peut toujours trouver $p$ fonctions abéliennes qui ne soient liées par aucune relation.

Soit en effet $F\left(v_{i}\right)$ une fonction abélienne quelconque; considérons avec M. Wirtinger les $p$ fonctions

$$
F_{k}\left(v_{i}\right)=F\left(v_{i}+\alpha_{i k}\right)
$$

les $\alpha$ étant des constantes quelconques. Si entre ces $p$ fonctions il $y$ avait une relation, leur déterminant fonctionnel serait nul, et comme les $\alpha_{i k}$ sont quelconques, le déterminant où le $i^{\text {e }}$ élément de la $k^{e}$ ligne est

$$
\frac{d}{d \alpha_{i k}} F\left(\alpha_{1 k}, \alpha_{2 k}, \ldots, \alpha_{p k}\right)
$$

serait identiquement nul quelles que soient les valeurs attribuées anx $p^{2}$ quantités $\alpha$. Si nous considérons les $p$ quantités

$$
u_{1}=\alpha_{11}, \quad u_{2}=\alpha_{21}, \ldots, u_{p}=\alpha_{p 1}
$$

comme des variables et les $p^{2}-p$ autres $\alpha$ comme des constantes, cela veut dire que la fonction $F\left(u_{1}, u_{2}, \ldots, u_{p}\right)$ satisfait à une équation de la forme

$$
A_{1} \frac{d F}{d u_{1}}+A_{2} \frac{d F}{d u_{2}}+\ldots+A_{p} \frac{d F}{d u_{p}}=0
$$

c'est à dire que $F$ ne dépend que de $p$ - I combinaisons linéaires des variables $u$, ce que nous ne supposerons pas. 


\section{§ 3. Démonstration du théorème $B$.}

Théorème B. Toute fonction $2 p$ fois périodique de $p$ variables peut s'exprimer par le moyen des fonctions $\boldsymbol{\theta}$.

Riemann paraît avoir connu ce théorème; en tout cas, Weierstrass l'avait démontré et je crois qu'il avait dû dans son cours exposer les principes de sa démonstration; quoi qu'il en soit, cette démonstration n'avait pas été publiée et ses élèves, s'ils l'avaient connue, ne l'avaient communiquée à personne. C'est ce qui nous décida, M. PiCARD et moi, à aborder la question et nous donnâmes une démonstration de ce théorème dans les Comptes Rendus en 1883 . Cette $1{ }^{\text {ère }}$ démonstration, dont nous reparlerons plus loin, était-elle identique à celle de Wererstrass, nous l'ignorions. Ce n'est que longtemps après, au moment de la publication des oeurres complètes $\mathrm{du}$ grand géomètre que nous avons su que les deux méthodes ne différaient pas essentiellement.

M. Appell donna ensuite (Journal de Lrouville 1891) une $2^{\mathrm{e}}$ démonstration du théorème $B$ entièrement différente de la ${ }_{1}{ }^{\text {ere }}$; puis vinrent deux autres démonstrations, l'une de M. PrCard (Comptes Rendus, tome I 24, page 1490) et l'autre de moi (Comptes Rendus, tome I 24, page I407. et Acta mathematica, tome 22).

Cette démonstration du tome 22 n'était autre chose qu'une adaptation à un but nouveau de la démonstration que j'avais donnée au tome 2 d'un théorème général sur les fonctions méromorphes; j’ai énoncé plus haut un théorème général d'après lequel toute fonction méromorphe est le quotient de deux fonctions entières et j'ai dit qu'outre ma démonstration, il en existe une autre qui a été développée par $M$. Cousin dans sa thèse (Acta mathematica, tome 19).

Eh bien, si ma démonstration peut être adaptée au théorème $B$, il en est probablement de même de celle de M. Cousis ce qui nous fournirait une cinquième démonstration du théorème $B$, entièrement différente des 4 premières. Cependant quand j'ai cherché à développer cette démonstration en lui conservant sa forme primitive, j'ai rencontré certaines difficultés. J'ai donc dû $\mathrm{y}$ introduire certaines modifications, et c'est cette démonstration modifiée, qui tient pour aussi dire le milieu entre celle de M. Cousin et la mienne que je vais chercher à adapter à l'étude du théorème 
B. Rappelons d'abord les hypothèses que nous faisons. Nous avons dans l'espace à $2 p$ dimensions une infinité de domaines

$$
D_{1}, D_{2}, \ldots
$$

tels que tout point de l'espace appartienne au moins à l'un de ces domaines et que dans le domaine $D_{i}$ par exemple, la fonction abélienne $F$ qu'il s'agit d'étudier soit égale à

$$
F=\frac{P_{i}}{Q_{i}}
$$

$P_{i}$ et $Q_{i}$ étant holomorphes dans le domaine $D_{i}$.

Nous pouvons supposer qu'en aucun point $M$ de $D_{i}$, les deux fonctions $P_{i}$ et $Q_{i}$ ne soient divisibles par une même fonction $H$, holomorphe dans le voisinage de $M$ et s'annulant en $M$, de telle façon que l'on ait:

$$
P_{i}=H P_{i}^{\prime}, \quad Q_{i}=H Q_{i}^{\prime},
$$

$P_{i}^{\prime}$ et $Q_{i}^{\prime}$ étant holomorphes dans le voisinage de $M$.

On pourrait dire que si cela avait lieu on diviserait $P_{i}$ et $Q_{i}$ par $H$, et que dans la partie $D_{i}^{\prime}$ du domaine $D_{i}$ où $H$ est holomorphe, on écrirait:

$$
F=\frac{P_{i}^{\prime}}{Q_{i}^{\prime}}
$$

au lieu de $F=\frac{P_{i}}{Q_{i}}$. Mais ce raisonnement serait insuffisant, parce qu'on pourrait craindre que dans le domaine $D_{i}^{\prime}$, qui est une partie de $D_{i}$, ne se trouvent des points où $P_{i}^{\prime}$ et $Q_{i}^{\prime}$ fussent eux-mêmes divisibles par une même fonction holomorphe, qu'on soit par conséquent obligé de détacher du domaine $D_{i}^{\prime}$ un domaine plus petit $D_{i}^{\prime \prime}$ où l'expression de $F$ devrait encore être modifiée, et qu'on ne soit forcé de poursuivre cette opération indéfiniment.

Nous raisonnerons donc de la manière suivante.

Soit $v_{i}=a_{i}$ un point situé dans le domaine $D_{k}$ et où

$$
P_{k}=\text { o. }
$$

De l'équation $P_{k}=0$, on pourra tirer $v_{1}$ par exemple en fonction algébroïde de $v_{2}, v_{3}, \ldots, v_{p}$. L'équation comportera d'ailleurs plusieurs solutions

$$
v_{1}=\varphi_{1}, \quad v_{1}=\varphi_{2}, \ldots, \quad v_{1}=\varphi_{q} ;
$$


$\varphi_{1}, \varphi_{2}, \ldots, \varphi_{q}$ étant des fonctions algébroïdes de $v_{2}, v_{8}, \ldots v_{p}$ se réduisant à $\alpha_{1}$ pour $v_{2}=\alpha_{2}, v_{3}=\alpha_{3}, \ldots, v_{p}=\alpha_{p}$.

Nous pourrons autour du point $v_{i}=\alpha_{i}$, trouver un domaine $\Delta_{k}$ intérieur à $D_{k}$ dans lequel les fonctions $\varphi$ restent algébroïdes et dans lequel enfin l'équation $P_{k}=$ o n'admette pas d'autre solution que les solutions (2).

Les fonctions algébroïdes $\varphi$ pourront se répartir en groupes de la façon suivante; si

$$
\left(v_{1}-\varphi_{1}\right)\left(v_{1}-\varphi_{2}\right) \ldots\left(v_{1}-\varphi_{m}\right)
$$

est une fonction holomorphe de $v_{1}, v_{2}, \ldots, v_{p}$ dans le voisinage de $v_{i}=\alpha_{i}$ (ce sera bien entendu un polynome entier d'ordre $m$ et $v_{1}$ ) et si cette fonction holomorphe est irréductible, c'est à dire n'est pas le produit de deux fonctions holomorphes de même forme, nous dirons que les fonctions $\varphi_{1}$, $\varphi_{2}, \ldots, \varphi_{m}$ forment un même groupe.

D'ailleurs les dimensions du domaine $\Delta_{k}$ restant finies quel que soit le point $v_{i}=\alpha_{i}$ nous pourrons diviser le domaine $D_{k}$ en un nombre fini de domaines analogues à $\Delta_{k}$.

Supposons maintenant qu'en faisant $v_{1}=\varphi_{1}$, on annule identiquement non seulement $P_{k}$ mais encore $Q_{k}$; il en sera évidemment de même quand on fera $v_{1}=\varphi_{2}, v_{1}=\varphi_{3}, \ldots, v_{1}=\varphi_{m}$; les fonctions $\varphi_{2}, \varphi_{3}, \ldots, \varphi_{m}$ étant les autres fonctions du même groupe. Supposons pour fixer les idées qư'il y ait un second groupe de fonctions $\varphi_{1}^{\prime}, \varphi_{2}^{\prime}, \ldots, \varphi_{n}^{\prime}$ qui substituées à la place de $v_{1}$ annulent identiquement $P_{k}$ et $Q_{k}$ et qu'il n'y en ait pas d'autre.

Alors $P_{k}$ et $Q_{k}$ seront divisibles dans le domaine $\Delta_{k}$ par la fonction holomorphe:

$$
H_{k}=\left(v_{1}-\varphi_{1}\right)\left(v_{1}-\varphi_{2}\right) \ldots\left(v_{1}-\varphi_{m}\right)\left(v_{1}-\varphi_{1}^{\prime}\right)\left(v_{1}-\varphi_{2}^{\prime}\right) \ldots\left(v_{1}-\varphi_{n}^{\prime}\right)
$$

de sorte qu'on aura

$$
P_{k}=H_{k} P_{k}^{\prime}, \quad Q_{k}=H_{k} Q_{k}^{\prime} ;
$$

les fonctions $H_{k}, P_{k}^{\prime}$ et $Q_{k}^{\prime}$ resteront holomorphes dans tout le domaine $\Delta_{k}$, et on aura dans le domaine $\boldsymbol{\Delta}_{k}$

$$
F=\frac{P_{k}^{\prime}}{Q_{k}^{\prime}}
$$

$P_{k}^{\prime}$ et $Q_{k}^{\prime}$ n'ayant plus de facteur commun s'annulant à l'intérieur de $\Delta_{k}$. En résumé nous pouvons toujours supposer que $P_{k}$ et $Q_{k}$ ne sont jamais divisibles par un même facteur $H$, holomorphe dans le voisinage d'un point intérieur à $D_{k}$ et s'annulant en ce point. 
Cela posé supposons que l'on ait $F=\frac{P_{i}}{Q_{i}}$ dans le domaine $D_{i}$ et $F=\frac{P_{k}}{Q_{k}}$ dans le domaine $D_{k}$. Si les deux domaines ont une partie commune, on aura dans cette partie commune

$$
\frac{P_{i}}{P_{k}}=\frac{Q_{i}}{Q_{k}}
$$

$P_{i}$ ne peut s'annuler sans que $P_{k}$ s'annule; car alors on aurait

$$
Q_{i}=\frac{P_{i}}{P_{k}} Q_{k}
$$

et $Q_{i}$ s'annulerait en ce même point. Alors $Q_{i}$ serait divisible par $P_{i}$, car $\frac{\mathrm{I}}{\boldsymbol{P}_{k}}$ est holomorphe puisque $P_{k}$ ne s'annule pas. Donc $P_{i}$ et $Q_{i}$ seraient divisibles par un même facteur $\boldsymbol{P}_{i}$ ce qui est contraire à l'hypothèse que nous venons de faire.

Donc $\frac{P_{k}}{P_{i}}$ ne peut devenir infini et par conséquent est holomorphe dans toute la partie commune aux deux domaines et il en est de même de $\frac{P_{i}}{P_{k}}$.

Maintenant la fonction $\boldsymbol{F}$ étant périodique, chaque prismatoïde des périodes sera divisé en un certain nombre de domaines $D_{k}$; et on pourra supposer que tous ces prismatoïdes sont divisés de la même manière, c'est à dire que si l'une d'eux comprend $n$ domaines

$$
D_{1}, D_{2}, \ldots, D_{n}
$$

l'autre comprendra $n$ domaines

$$
D_{1}^{\prime}, D_{2}^{\prime}, \ldots, D_{n}^{\prime}
$$

correspondant respectivement ̀̀ $D_{1}, D_{2}, \ldots, D_{n}$, de telle façon que l'on passe d'un domaine au domaine correspondant en augmentant les variables d'une période.

De plus, dans deux domaines correspondants $D_{k}$ et $D_{k}^{\prime}$ les fonctions $P$ et $Q$ reprendront les mêmes valeurs; de telle sorte que l'on aura dans $D_{k}$ :

$$
F=\frac{P_{k}}{Q_{k}}
$$


et dans $D_{k}^{\prime}$ :

$$
F=\frac{P_{k}^{\prime}}{Q_{k}^{\prime}}
$$

et que

$$
\begin{aligned}
& P_{k}^{\prime}\left(v_{1}, v_{2}, \ldots, v_{p}\right)=P_{k}\left(v_{1}+\beta_{1}, v_{2}+\beta_{2}, \ldots, v_{p}+\beta_{p}\right), \\
& Q_{k}^{\prime}\left(v_{1}, v_{2}, \ldots, v_{p}\right)=Q_{k}\left(v_{1}+\beta_{1}, v_{2}+\beta_{2}, \ldots, v_{p}+\beta_{p}\right)
\end{aligned}
$$

$\beta_{1}, \beta_{2}, \ldots, \beta_{p}$ étant la période par laquelle on passe de $D_{k}$ et $D_{k}^{\prime}$.

Les domaines $D_{1}, D_{2}$ etc. empiètent les uns sur les autres, mais je puis considérer une infinité de domaines $\Delta_{1}, \Delta_{2}$, etc. de telle façon: $\mathrm{I}^{\circ}$ que le domaine $\Delta_{k}$ soit intérieur à $D_{k} ; 2^{\circ}$ que tout point de l'espace appartienne à un des domaines $\Delta$ et à un seul, à moins qu'il n'appartienne à la frontière qui sépare deux de ces domaines.

Il est clair que les domaines $\Delta$ restent encore arbitraires dans une certaine mesure et que je puis leur faire subir de petites déformations pourvu que $\Delta_{k}$ reste intérieur à $D_{k}$.

Considérons deux domaines $D_{n}$ et $D_{q}$ ayant une partie commune; nous aurons respectivement dans ces deux domaines

$$
F=\frac{P_{n}}{Q_{n}}, \quad F=\frac{P_{q}}{Q_{q}} .
$$

Soient $\Delta_{n}$ et $\Delta_{q}$ les domaines $\Delta$ correspondant à $D_{n}$ et à $D_{q}$ et soit $S_{n q}$ la frontière qui sépare $\Delta_{n}$ de $\Delta_{q}$.

$\mathrm{Je}_{\mathrm{e}}$ considère une fonction $\Phi$ définie de la façon suivante. Dans le domaine $\Delta_{k}$ on aura:

$$
\Phi=\log P_{k} .
$$

Ce n'est pas une fonction qui jouisse de la continuité analytique, car quand en franchissant $S_{n q}$ on passe de $\Delta_{n}$ dans $\Delta_{q}$, cette fonction subit un saut brusque égal à

$$
\log \frac{P_{q}}{P_{n}}
$$

On peut dire encore que si l'on appelle $\phi^{\prime}$ la continuation analytique de $\Phi$ au delà de $S_{n q}$ quand ayant franchi cette coupure on a passé de $\Delta_{n}$ dans $\Delta_{q}$, on aura dans $\Delta_{q}$ :

$$
\Phi-\Phi^{\prime}=\log \frac{P_{q}}{P_{n}}
$$


Ce qui nous intéresse c'est que dans la partie commune à $D_{n}$ et à $D_{q}$ cette fonction $\log \frac{P_{q}}{P_{n}}$ sera holomorphe, car le rapport de $P_{q}$ à $P_{n}$ ne peut ni s'annuler ni devenir infini.

Posons

$$
v_{k}=x_{k}+i y_{k}
$$

de telle façon que les parties réelles et imaginaires, c'est à dire les $x$ et les $y$ soient les coordonnées d'un point dans notre espace à $2 p$ dimensions.

Soit $M^{\prime}$ un point situé sur la variété $S_{n q}$ qui a $2 p-$ I dimensions, et dont les coordonnées seront $x_{k}^{\prime}$ et $y_{k}^{\prime}$.

Soit $M$ le point de coordonnées courantes $x_{k}$ et $y_{k}$. Soit

$$
r=\sqrt{2\left(x-x_{k}^{\prime}\right)^{2}+2\left(y-y_{k}^{\prime}\right)^{8}}
$$

la distance de ces deux points. Soit $d \omega^{\prime}$ un élément de la variété $S_{n q}$ ayant pour centre de gravité le point $\boldsymbol{M}^{\prime}$. Soit $\delta^{\prime}$ une fonction quelconque des coordonnées du point $M^{\prime}$ et envisageons l'intégrale

$$
V=\int \frac{\delta^{\prime} d \omega^{\prime}}{r^{2 p-2}}
$$

étendue à tous les éléments $d \omega^{\prime}$ de la variété $S_{n q}$. C'est ce qu'on appelle le potentiel d'une simple couche. Pour tous les points situés en dehors de $S_{n q}$, c'est une fonction holomorphe $\operatorname{des} x$ et des $y$; cette fonction reste finie ainsi que ses dérivées quand le point $M$ vient sur $S_{n q}$. Si la variété $S_{n q}$ est analytique et si d'autre part $\delta^{\prime}$ est une fonction analytique des coordonnées de $M^{\prime}$, il arrivera que la fonction $V$ pourra être continuée analytiquement au delà de la coupure $S_{n q}$. Soit alors $M_{1}$ et $M_{2}$ deux points voisins l'un de l'autre, mais situés de part et d'autre de cette coupure. Soient $V_{1}$ et $V_{2}$ les valeurs de l'intégrale $V$ en ces deux points; soit $V^{\prime}$ la continuation analytique de la fonction $V$ quand partant du point $M_{1}$ on franchit la coupure et soit $V_{2}^{\prime}$ la valeur de $V^{\prime}$ en $M_{2}$.

Alors $V_{2}^{\prime}$ comme $V_{2}$ seront des fonctions analytiques des coordonnées de $M_{2}$, mais $V_{2}^{\prime}$ ne sera pas en général égal à $V_{2}$. Nous devons observer que dans le cas d'une simple couche, la fonction $V$ ne subit pas un saut brusque quand on franchit la coupure; de sorte que sur la coupure même

$$
V_{2}=V_{2}^{\prime} \text {. }
$$


Il n'en est pas de même des dérivées, et si l'on représente en particulier par $\frac{d V}{d n}$ la dérivée estimée suivant la normale à la coupure $S_{n q}$, la différence

$$
\frac{d V_{z}}{d n}-\frac{d V_{2}^{\prime}}{d n}
$$

sur la coupure même ne sera pas nulle, mais elle sera égale à $\grave{o}^{\prime}$ à un facteur constant près qui ne dépendra que du nombre $2 p$ des dimensions.

Soit maintenant $\varepsilon^{\prime}$ une autre fonction analytique des coordonnées de $\boldsymbol{M}^{\prime}$, et considérons l'intégrale

$$
W=\int \varepsilon^{\prime} d \omega^{\prime} \frac{d r^{2-2 p}}{d n},
$$

e'est ce qu'on appelle le potentiel d'une double couche. Alors si nous appelons encore $W_{1}$ et $W_{2}$ les valeurs de $W$ aux points $M_{1}$ et $M_{2}$, nous verrons que $W_{1}$ est une fonction analytique des coordonnées de $M_{1}$, comme $W_{2}$ de celles de $M_{2}$. De plus $W$ peut être continué analytiquement au delà de la coupure, et si l'on appelle $W^{\prime}$ cette continuation et $W_{2}^{\prime}$ la valeur de $W^{\prime}$ en $M_{2}$, alors $W_{2}^{\prime}$ sera fonction analytique de $M_{2}$.

Sur la coupure même on aura $\frac{d W_{9}}{d n}=\frac{d W_{2}^{\prime}}{d n}$, mais la différence $W_{2}-W_{2}^{\prime}$ ne sera pas nulle, elle sera égale à $\varepsilon^{\prime}$ à un facteur constant près ne dépendant que du nombre $2 p$ des dimensions.

Nous reprendrons maintenant notre fonction holomorphe

$$
\Phi-\Phi^{\prime}=\log \frac{P_{q}}{P_{n}}
$$

et nous considérerons sa partie réelle que j'appellerai $R_{n q}$

$$
R_{n q}=\log \left|\frac{P_{q}}{P_{n}}\right|
$$

Nous prendrons alors:

$$
\delta^{\prime}=\frac{d R_{* q}}{d n}, \quad \varepsilon^{\prime}=R_{n q}
$$

et j'envisagerai l'intégrale

$$
J_{n q}=a V+b W
$$

les $a$ et les $b$ étant des constantes. 
J'appellerai $J_{n q}^{\prime}$ le prolongement analytique de $J_{n q}$ par delà la coupure. $\mathrm{Si}$ les coefficients constants $a$ et $b$ sont convenablement choisis (c'est à dire s'ils sont les inverses des coefficients constants dont je parlais tout à l'heure et qui ne dépendent que du nombre $2 p$ des dimensions), nous aurons:

$$
J_{n q}^{\prime}-J_{n q}=R_{n q} \text {. }
$$

Soit en effet $\psi$ une fonction qui soit égale à $J_{n q}$ d'un côté de la coupure et à $J_{n q}+R_{n q}$ de l'autre côté de la coupure. Des deux côtés de la coupure, $\psi$ sera une fonction harmonique des $2 p$ variables $x$ et $y$; je veux dire par là qu'elle sera holomorphe et satisfera à l'équation de LAPIACE $\Delta \psi^{\prime}=0$; nous ne savons pas encore s'il en sera de même sur la coupure même; mais sur cette coupure la fonction $\Psi$ est continue (si nos coefficients $a$ et $b$ sont convenablement choisis) et il en est de même de $\frac{d \Psi}{d n}$. On en conclut que la fonction $\psi$ reste holomorphe sur la coupure même et que $J_{n q}+R_{n q}$ est la continuation analytique de $J_{n q}$.

$\mathrm{Si}$ donc on a une fonction qui soit égale à $\log \left|P_{q}\right|-J_{n q}$ dans le domaine $\Delta_{q}$ et à $\log \left|P_{n}\right|-J_{n q}$ dans le domaine $\Delta_{n}$ cette fonction restera analytiquement continue quand on franchira la coupure $S_{n q}$; elle sera harmonique dans l'ensemble des deux domaines $\Delta_{q}$ et $\Delta_{n}$; (sauf pour les points de $\Delta_{n}$ ou de $\Delta_{q}$ où $P_{n}$ ou $P_{q}$ s'annulent; en ces points c'est la différence $\Psi-\log \left|P_{n}\right|$ ou $\Psi-\log \left|P_{q}\right|$ qui est harmonique).

L’intégrale $J_{n q}$ joue le rôle que joue dans la démonstration de M. Cousin l'intégrale de CAUCHY

$$
\frac{1}{2 \pi i} \int \frac{\left(f_{n}-f_{p}\right) d z}{z-y}
$$

mais c'est une intégrale multiple.

Une propriété importante rapproche encore notre intégrale de celle de Cauchy. Soit $S_{n q}^{\prime}$ une coupure peu différente de $S_{n q}$; je suppose que la frontière complète de $S_{n q}^{\prime}$ soit la même que celle de $S_{n q}$ de façon que l'ensemble des deux coupures forme une variété fermée enfermant un certain domaine $\Delta^{\prime}$. Soit $K_{n q}$ la même intégrale que $J_{n q}$ mais prise le long de $S_{n q}^{\prime}$. On aura alors en dehors de $\Delta^{\prime}$ :

et à l'intérieur de $\Delta^{\prime}$ :

$$
J_{n q}=K_{n q}
$$

$$
J_{n q}=K_{n q}+R_{n q}
$$


Il en résulte que si $\psi$ est une fonction égale à $\log \left|P_{q}\right|-J_{n q}$ dans $\Delta_{q}$ et à $\log \left|P_{n}\right|-J_{n q}$ dans $\Delta_{n}$ et si d'autre part $\psi^{\prime}$ est une fonction égale à $\log \left|P_{q}\right|-K_{n q}$ dans le domaine $\Delta_{q}+\Delta^{\prime}$, à $\log \left|P_{n}\right|-K_{n q}$ dans le domaine $\Delta_{n}-\Delta^{\prime}$, on aura

$$
\psi=\phi^{\prime}
$$

En d'autres termes on aura pu déplacer et déformer un peu la coupure $S_{n q}$ sans changer la fonction $\psi$.

Cela posé, considérons quelques-uns de nos domaines en nombre fini

$$
\Delta_{1}, \Delta_{2}, \ldots, \Delta_{q}
$$

et les coupures qui séparent ces domaines (2). Soit $\psi$ une fonction qui est égale dans $\Delta_{n}\left(\Delta_{n}\right.$ étant l'un des domaines (2)) à:

$$
\phi=\log \left|P_{n}\right|-\Sigma J_{n q}
$$

la sommation étant étendue à toutes les intégrales $J_{n q}$ relatives aux coupures qui séparent les domaines (2).

La fonction $\psi$ est harmonique, dans l'ensemble des domaines (2) sauf peut-être pour les points qui appartiennent à plus de deux de ces domaines; (et sauf certainement pour les points où l'un des $P_{n}$ s'annule, car en ce points e'est $\psi-\log \left|P_{n}\right|$ qui est harmonique).

Soit done $N$ un des points qui appartiennent à plus de deux des domaines (2). De ce point comme centre décrivons une hypersphère $H$; déformons ensuite les coupures de façon qu'elles ne changent pas ni en dehors de l'hypersphère, ni sur l'hypersphère elle-même, mais seulement dans l'intérieur de l'hypersphère et qu'après la déformation, le point $N$ ne soit plus sur une coupure.

Je dis que la fonction $\phi$ n'a pas changé. Nous avons vu plus haut que cette fonction ne change pas quand une des coupures est déformée sans que sa frontière complète varie. Ici les choses ne se passent pas tout à fait comme cela, puisque le point $N$ se trouvant sur la frontière commune de plusieurs coupures, nous sommes obligés de déformer ces frontières si nous voulons que le point $N$ cesse d'être sur une coupure. C'est seulement en dehors de l'hypersphère $H$ que les frontières ne varient pas.

Soit alors $S_{n q}$ l'une des coupures, $S_{n q}^{\prime}$ la coupure déformée; ce sont Acta mathematica. 26. Imprimé le 11 avril 1902. 
des variétés à $2 p-$ I dimensions; soit $F$ la frontière de $S_{n q}, F^{\prime}$ celle de $S_{n q}^{\prime}$; ce sont des variétés à $2 p-2$ dimensions; quand pendant sa déformation continue, cette frontière va de sa position initiale $F$ à sa position finale $F^{\prime}$, elle engendre une variété à $2 p-$ I dimensions que j'appelle $T_{n q}$. Alors la frontière de $T_{n q}$ est formée de $F$ et de $F^{\prime}$; et la variété $S_{n q}^{\prime \prime}=S_{n q}^{\prime}+T_{n q}$ a pour frontière $F$, c'est à dire même frontière que $S_{n q}$.

La fonction $\psi$ ne change donc pas quand on remplace les coupures $S_{n q}$ par les coupures $S_{n q}^{\prime \prime}$. Qu'est ce à dire? Soit $C$ une variété à $2 p-2$ dimensions appartenant à plus de deux domaines (2); par exemple aux domaines $\Delta_{1}, \Delta_{2}, \ldots, \Delta_{h}$; ces domaines doivent être contigus deux à deux, par exemple $\Delta_{1}$ à $\Delta_{2}, \Delta_{2}$ à $\Delta_{3}, \ldots, \Delta_{h-1}$ à $\Delta_{h}, \Delta_{h}$ à $\Delta_{1}$. Alors $C$ fera partie de la frontière de $h$ coupures, à savoir $S_{12}, S_{23}, \ldots, S_{h-1 . h}, S_{h .1}$. Pendant la déformation, $C$ ira de sa position initiale $C$ à sa position finale $C^{\prime}$ et engendrera ainsi une variété $U$ à $2 p$ - I dimensions. D'après sa définition même, $U$ fera partie de $T_{1.2}, T_{2.3}, \ldots, T_{h .1}$.

Cela posé si nous appelons $\psi^{\prime}$ et $\psi^{\prime \prime}$ ce que devient $\psi$ quand on remplace les coupures $S_{n q}$ par les coupures $S_{n q}^{\prime}$ et $S_{n q}^{\prime \prime}$; nous avons déjà vu que $\psi=\phi^{\prime \prime}$. Quelle est la différence entre $\psi^{\prime}$ et $\psi^{\prime \prime}$. Ces deux fonctions ne peuvent différer que par les intégrales $j_{n q}$ c'est à dire par des intégrales de même forme que les $J_{n q}$ mais prises le long des variétés $T_{n q}$, nous aurons donc:

$$
\psi^{\prime \prime}-\psi^{\prime}=\Sigma j_{n q}
$$

la sommation étant étendue à tous les $j_{n q}$, c'est à dire à tous les $T_{n q}$, mais nous pouvons écrire

$$
j_{n q}=\Sigma j_{n q}^{\prime}
$$

en appelant $j_{n q}^{\prime}$ la même intégrale étendue à l'une des variétés telles que $U$ dont se compose $T_{n q}$. $\Pi$ vient alors:

$$
\phi^{\prime \prime}-\phi^{\prime}=\Sigma \Sigma j_{n q}^{\prime},
$$

l'un des signes $\Sigma$ se rapportant aux différentes variétés telles que $U$ et l'autre aux différents $T_{n q}$ dont une de ces variétés fait partie. Or si je considère l'une de ces variétés, par exemple $U$, et que j'étende la sommation à tous les $T_{n q}$ dont $U$ fait partie, c'est à dire à $T_{12}, T_{23}, \ldots, T_{h .1}$, il viendra:

$$
\Sigma j_{n q}^{\prime}=j_{12}^{\prime}+j_{2.2}^{\prime}+\ldots+j_{h .1}^{\prime} .
$$


Nous nous rappelons comment a été définie l'intégrale $J_{n q}$ et le rôle que jouait la fonction que j’ai appelée $R_{n q}$. Nous voyons que l'intégrale totale $\Sigma j_{n q}^{\prime}$ se calculera de la même manière en remplaçant $R_{n q}$ par

$$
R_{1.2}+R_{2.3}+\ldots+R_{h .1}
$$

l'intégration étant d'ailleurs étendue à $U$. Mais on a:

$$
R_{12}+R_{23}+\ldots+R_{h .1}=\log \left|\frac{P_{2}}{P_{1}}\right|+\log \left|\frac{P_{3}}{P_{2}}\right|+\ldots+\log \left|\frac{P_{1}}{P_{h}}\right|=0 .
$$

Donc on aura

$$
\psi^{\prime}-\phi^{\prime \prime}=0, \quad \phi=\psi^{\prime}
$$

C. Q. F. D.

Or le point $N$ n'est pas un point singulier pour $\psi^{\prime}$ puisqu'il n'est pas sur les coupures $S^{\prime}$; ce n'est donc pas non plus un point singulier pour $\phi$.

D'où nous concluons que $\phi$ est harmonique dans l'ensemble des domaines (2), sauf pour les points de $D_{n}$ où $P_{n}$ s'annule et où c'est $\psi-\log \left|P_{n}\right|$ qui est harmonique.

Reprenons les intégrales $V, W, J_{n q}$; on se rappelle le rôle que jouait dans la définition de ces intégrales la fonction

$$
r^{2-2 p}
$$

qui dépend des $x$, des $y$, des $x^{\prime}$ et des $y^{\prime}$. Développons-la suivant les puissances des $x$ et des $y$ et soit.

$$
r^{2-2 p}=\rho_{0}+\rho^{\prime}
$$

où $\rho_{0}$ représente l'ensemble des termes de degré $O$, I ou 2 par rapport aux $x$ et aux $y$ et $\rho^{\prime}$ l'ensemble des termes de degré supérieur.

Soit maintenant $L_{n q}$ une intégrale analogue à $J_{n q}$, mais où $r^{2-2 p}$ est remplacé par $\rho^{\prime}$; soit maintenant $\chi$ une fonction définie comme $\psi$, mais où les $J_{n q}$ sont remplacées par les $L_{n q}$, de telle façon que dans $\Delta_{n}$ on ait:

$$
\chi=\log \left|P_{\mathrm{n}}\right|-\Sigma L_{\mathrm{n} q} ;
$$

la fonction $\chi$ jouira des mêmes propriétés que la fonction $\psi$, dont elle ne différera d'ailleurs que par un polynome du $2^{\text {d }}$ degré en $x$ et $y$.

Mais supposons que l'on prenne un nombre de plus en plus grand de domaines (2) de façon que l'ensemble de ces domaines tende à embrasser 
l'espace tout entier; de sorte enfin qu'à la limite la fonction $\chi$ (resp. $\left.\chi-\log \left|P_{n}\right|\right)$ soit harmonique dans tout l'espace.

Pour que ce passage à la limite soit légitime, il faut que la série $\Sigma L_{n q}$ converge; or c'est ce qu'il est aisé de constater, tandis que la série $\Sigma J_{n q}$ aurait été divergente. Nous n'avons qu'à reproduire ici la démonstration du tome 22 (pages $167, \mathrm{I} 68$ ). La fonction $\chi$ jouissant d'ailleurs des mêmes propriétés que la fonction $V$ du tome 22 , la démonstration s'achèverait de la même manière.

\section{\$ 4. Autre forme de la démonstration.}

Mais on aura avantage à mettre la démonstration sous une forme un peu différente.

Nous pourrons supposer d'abord que dans un certain domaine $D$, assez grand pour qu'un prismatoïde des périodes $\Pi$ y soit contenu tout entier, notre fonction abélienne $F$ peut être mise sous la forme du quotient $\frac{P}{Q}$ de deux séries entières convergeant dans tout le domaine $D$.

Cela peut s'établir aisément en partant des hypothèses du paragraphe précédent, et cela de plusieurs manières, soit par la méthode de M. Cousin, soit pas ma méthode du tome 2, soit par la méthode mixte du paragraphe précédent.

D'ailleurs $P$ et $Q$ n'auront pas de facteur commun s'annulant à l'intérieur de $D$.

$\mathrm{Si}$ nous augmentons nos variables d'une période fondamentale $a_{i}$, $P\left(v_{i}\right)$ et $Q\left(v_{i}\right)$ deviendront $P\left(v_{i}+a_{i}\right)$ et $Q\left(v_{i}+a_{i}\right)$. Les séries $P\left(v_{i}\right)$ et $Q\left(v_{i}\right)$ convergent dans le domaine $D$; les séries $P\left(v_{i}-a_{i}\right)$ et $Q\left(v_{i}-a_{i}\right)$ convergeront dans un domaine $D^{\prime}$ que l'on obtiendra en faisant subir à $D$ une translation représentée en grandeur et direction par la période $a_{i}$. Les deux domaines $D$ et $D^{\prime}$ ont une partie commune puisque $D$ est plus grand qu'un prismatoïde des périodes. Dans cette partie commune, les deux séries $P\left(v_{i}\right)$ et $P\left(v_{i}+a_{i}\right)$ convergent et leur rapport

$$
\frac{P\left(v_{i}\right)}{P\left(v_{i}-a_{i}\right)}
$$


ne peut devenir ni nulle ni infinie. Si en effet il s'annulait par exemple, les deux séries

$$
\boldsymbol{P}\left(v_{i}\right), Q\left(v_{i}\right)=P\left(v_{i}\right) \frac{Q\left(v_{i}-a_{i}\right)}{P\left(v_{i}-a_{i}\right)}
$$

auraient un facteur commun $\boldsymbol{P}\left(v_{i}\right)$ s'annulant à l'intérieur de $D$.

Nous allons maintenant définir une fonction auxiliaire que nous appellerons $W$. Soient $M$ et $M^{\prime}$ deux points ayant pour coordonnées, le $\mathrm{I}^{\mathrm{er}} x_{k}, y_{k}$, le $2^{\mathrm{d}} x_{k}^{\prime}, y_{k}^{\prime} ;$ soit

leur distance.

$$
r=\sqrt{\Sigma\left(x_{k}-x_{k}^{\prime}\right)^{2}+\Sigma\left(y_{k}-y_{k}^{\prime}\right)^{2}}
$$

Soit $M^{\prime \prime}$ un point ayant pour coordonnée $x_{k}^{\prime}+b_{k}^{\prime}, y_{k}^{\prime}+c_{k}^{\prime}$ en désignant par $b_{k}^{\prime}$ et $c_{k}^{\prime}$ les parties réelle et imaginaire d'une des périodes $a_{k}^{\prime}$ de notre fonction abélienne (je veux dire, soit d'une période fondamentale, soit d'une des combinaisons en nombre infini de ces périodes fondamentales c'est à dire d'une période quelconque).

Soit $\rho$ la distance des points $M$ et $M^{\prime \prime}$.

On sait que $r^{2-2 p}$ est une fonction harmonique des $x$ et des $y$, et il en est évidemment de même de $\rho^{2-2 p}$. Supposons qu'on développe $\rho^{2-2 p}$ suivant les puissances des $x$ et $\operatorname{des} y$ et que l'on écrive:

$$
\rho^{2-2 p}=\sigma+\tau
$$

en représentant par $\sigma$ l'ensemble des termes de degré $O$, I et 2 et par $\tau$ l'ensemble des termes de degré supérieur. En envisageant la période zéro, on aura en particulier

$$
r^{2-2 p}=\sigma_{0}+\tau_{0}
$$

Il est clair que $\tau$ est encore une fonction harmonique. Nous poserons alors:

$$
W=\Sigma \tau
$$

la sommation étant étendue à toutes les périodes, en y comprenant la période zéro.

Il est aisé de voir que la série $\Sigma \tau$ converge uniformément et on en conclut que $W$ est une fonction harmonique des $x$ et des $y$ satisfaisant à l'équation de Laplace $\Delta W=0$, sauf quand le point $M$ se confond avec le point $M^{\prime \prime}$, c'est à dire quand le vecteur $\boldsymbol{M} \boldsymbol{M}^{\prime}$ représente une période quelconque en grandeur et direction. 
Soient maintenant $\varphi$ et $\psi$ deux faces» opposées du prismatoïde de $I I$; soit $a_{i}$ la période fondamentale correspondante de telle sorte qu'on passe de $\psi$ à $\varphi$ en changeant $v_{i}$ en $v_{i}+\iota_{i}$. La face $\varphi$ appartient alors à la fois aux domaines $D$ et $D^{\prime}$. Dans la partie commune à ces deux domaines, qui comprend la face $\varphi$, la fonction

$$
\log P\left(v_{i}-a_{i}\right)-\log P\left(v_{i}\right)
$$

est holomorphe ainsi que nous l'avons vu. Nous appellerons $U+i V$ cette fonction holomorphe, et sa partie réelle $U$ sera évidemment une fonction harmonique des $x$ et des $y$.

Soit $d \omega^{\prime}$ un élément de la face $\varphi$ et supposons que le point $M^{\prime}$ défini plus hant soit au centre de gravité de $d \omega^{\prime}$. Soit $U^{\prime}$ la valeur de $U$ au point $M^{\prime}$.

Considérons alors l'intégrale

$$
J=\int\left(W \frac{d U^{\prime}}{d n}-\frac{d W}{d n} U^{\prime}\right) d \omega^{\prime}
$$

étendue à tous les éléments $d \omega^{\prime}$ de la face $\varphi$. Je désigne, bien entendu, par $\frac{d U^{\prime}}{d n} d n$ et $\frac{d W}{d n} d n$ les accroissements que subissent les fonctions $U^{\prime}$ et $W$ quand le point $M^{\prime}$ subit une déplacement $d n$, normalement à la face $\varphi$.

Il est clair que $J$ est une fonction harmonique $\operatorname{des} x$ et des $y$, sauf quand le point $M$ se trouve sur la face $\varphi$ ou sur l'une de ses transformées par l'addition d'une période. Et en effet quand le point $M$ coïncide avec le point $M^{\prime}$ (qui est sur la face $\varphi$ ) ou avec l'un des points $M^{\prime \prime}$, (c'est à dire avec un des transformés de $M^{\prime}$ pār l'addition d'une période), la fonction $W$ devient infinie.

La face $\varphi$ jointe à ses transformées par l'addition d'une période forme une suite indéfinie de variétés planes à $2 p$ - I dimensions, parallèles et équidistantes. Soient $S$ ces variétés.

Alors la fonction $J$ est analytique, sauf quand on franchit l'un des $S$. Qu'arrive-t-il maintenant quand le point $M$ franchit l'un des $S$ ? Soit $N^{\prime \prime}$ un point de l'un des $S$, et $N^{\prime}$ le point correspondant de $\varphi$. Déformons alors légèrement la face $\varphi$ dans la partie voisine du point $N^{\prime}$ et soit $\varphi^{\prime}$ le résultat de la déformation. Nous pourrons supposer que $N^{\prime}$ n'est pas sur $\varphi^{\prime}$, et que toute la partie de $\varphi$ qui n'est pas voisine de $N^{\prime}$ n'a pas subi de déformation et coïncide par conséquent avec la partie correspondante de $\varphi^{\prime}$. 
Alors entre $\varphi$ et $\varphi^{\prime}$ il $\mathrm{y}$ aura un petit domaine $\Delta$ dont la frontière se composera de la partie de $\varphi$ qui n'appartient pas à $\varphi^{\prime}$ et de la partie de $\varphi^{\prime}$ qui n'appartient pas à $\varphi$.

Nous considérerons également les transformés de $\varphi$, de $\varphi^{\prime}$ et de $\Delta$ par l'addition d'une période. Le point $N^{\prime \prime}$ se tronve sur l'un des transformés de $\varphi$, mais non pas sur l'un des transformés de $\varphi^{\prime}$; il est donc sur la frontière de l'un des transformés de $\Delta$, que j'appellerai $\Delta^{\prime}$.

Cela posé, considérons l'intégrale $J^{\prime}$ qui est formée comme $J$, mais étendue à $\varphi^{\prime}$ et non plus à $\varphi$. Elle sera analytique sauf quand le point $M$ sera sur $\varphi^{\prime}$ ou sur l'un de ses transformés.

La différence des deux intégrales est égale à

$$
J-J^{\prime}=\int\left(W \frac{d U^{\prime}}{d n}-\frac{d W}{d n} U^{\prime}\right) d \omega^{\prime}
$$

l'intégration étant étendue à la frontière du domaine $\Delta$. Elle reste analytique sauf quand le point $M$ est sur la frontière de $\Delta$ ou de l'un de ses transformés. Voyons par exemple ce qui se passe quand le point $M$ est voisin de la frontière de $\Delta^{\prime}$ et plus particulièrement voisin de $N^{\prime \prime}$.

Quand le point $M$ vient en $N^{\prime \prime}$ et le point $M^{\prime}$ en $N^{\prime}$, la fonction $W=\Sigma \tau$ devient infinie mais un seulement des termes de la somme $\Sigma \tau$ devient infini; soit $\rho_{1}^{2-2 p}$ le $\rho^{2-2 p}$ correspondant. Alors $W-\rho_{1}^{2-2 p}$ reste finie. Posons:

$$
W-\rho_{1}^{2-2 p}=H \text {. }
$$

Nous aurons:

$$
J-J^{\prime}=K+K_{1}
$$

avec

$$
\begin{aligned}
& K=\int\left(H \frac{d U^{\prime}}{d n}-U^{\prime} \frac{d H}{d n}\right) d \omega^{\prime} \\
& K_{1}=\int\left(\rho_{1}^{2-2 p} \frac{d U^{\prime}}{d u}-U^{\prime} \frac{d \rho_{1}^{2-2 p}}{d n}\right) d \omega^{\prime}
\end{aligned}
$$

les deux intégrales étant étendues à la frontière de $\Delta$.

La fonction $H$ est une fonction harmonique, $\operatorname{des} x^{\prime}$ et des $y^{\prime}$ (nous avons déjà vu qu'elle est harmonique par rapport aux $x$ et aux $y$, mais 
je la considère maintenant comme fonction des $x^{\prime}$ et des $y^{\prime}$ ). Elle reste analytique à l'intérieur de $\Delta$, que le point $M$ soit intérieur ou extérieur à $\Delta^{\prime}$ ou qu'il soit sur la frontière de $\Delta^{\prime}$, pourvu toutefois que ce point $M$, restant voisin de $\Delta^{\prime}$ comme nous le supposons, ne soit intérieur à aucun des autres transformés de $\Delta$.

De même la fonction $U^{\prime}$ est harmonique et analytique à l'intérieur de $\Delta$. Dans ces conditions le théorème de Gresn nous enseigne que l'intégrale $K$ est nulle. En ce qui concerne l'intégrale $K_{1}$, ce même théorème nous enseigne qu'elle est nulle si le point $M$ est extérieur à $\Delta^{\prime}$. Si le point $M$ est intérieur à $\Delta^{\prime}$, notre intégrale $K_{1}$ est égale, toujours d'après le même théorème, à

$$
\alpha U_{0}
$$

$\alpha$ étant un coefficient numérique qui ne dépend que du nombre $2 p$ des dimensions et $U_{0}$ la valeur que prend $U^{\prime}$ quand la distance $\rho_{1}$ s'annule.

Or quand la distance $\rho_{1}$ s'annule, e'est que le point $M^{\prime}$ vient au point de l'intérieur de $\Delta$ qui correspond à la position du point $M$ à l'intérieur de $\Delta^{\prime}$. Or soit $a_{k}^{\prime}=b_{k}^{\prime}+i c_{k}^{\prime}$ la période dont l'addition transforme le point $N^{\prime}$ en $N^{\prime \prime}$ et $\Delta$ en $\Delta^{\prime}$. Quand les points $M$ et $M^{\prime}$ occuperont les positions que j'ai dites, on aura

$$
x_{k}=x_{k}^{\prime}+b_{k}^{\prime}, \quad y_{k}=y_{k}^{\prime}+c_{k}^{\prime} ;
$$

on aura done

$$
U_{0}=U\left(x_{k}-b_{k}^{\prime}, y_{k}-c_{k}^{\prime}\right)
$$

et

$$
K_{1}=\alpha U\left(x_{k}-b_{k}^{\prime}, y_{k}-c_{k}^{\prime}\right) .
$$

Donc quand $M$ est extérieur à $\Delta^{\prime}$, on a

$$
J=J^{\prime}
$$

Quand le point $M$ franchit la coupure $S$ dans le voisinage de $N^{\prime \prime}$, pour pénétrer dans $\Delta$, l'intégrale $J$ n'est pas continue, mais l'intégrale $J^{\prime}$ l'est. Donc $J^{\prime}$ est la continuation analytique de $J$ au delà de la coupure $S$.

Quand $M$ est intérieur à $\Delta^{\prime}$, on a :

$$
J^{\prime}=J-a J\left(x_{l}-b_{k}^{\prime}, \eta_{k}-c_{k}^{\prime}\right) \text {. }
$$


Donc la continuation analytique de $J$ au delà de la coupure est

$$
J-\alpha U\left(x_{k}-b_{k}^{\prime}, y_{k}-c_{k}^{\prime}\right) \text {. }
$$

C'est la conclusion finale à laquelle je voulais arriver.

Le prismatoide a $2 p$ couples de faces opposées, $\varphi_{1}$ et $\phi_{1}, \varphi_{2}$ et $\psi_{2}, \ldots$, $\varphi_{2 p}$ et $\psi_{2 p}$. Soient $U_{q}$ et $J_{q}$ la fonction analogue à $U$ et l'intégrale analogue à $J$, relatives à la face $\varphi_{q}$.

Soit $\Phi$ une fonction qui dans le prismatoide $\Pi$ est égale à

$$
\log \left|P\left(v_{i}\right)\right|
$$

et qui dans le prismatoïde $\eta^{\prime}$ transformé de $\Pi$ par l'addition de la période $a_{k}^{\prime}=b_{k}^{\prime}+i c_{k}^{\prime}$ est égale à :

$$
\log \left|P\left(v_{i}-a_{i}\right)\right| \text {. }
$$

La fonction $\Phi$ est donc harmonique dans chacun des prismatoïdes, sauf aux points pour lesquels $F$ s'annule ou devient indéterminée. Mais elle est discontinue quand on passe d'un prismatö̈de dans l'autre. De plus elle est périodique par définition.

$\mathrm{Si}$ nous désignons par $a_{i q}$ la période fondamentale qui correspond à la face $\varphi_{q}$ et par $b_{i q}$ et $c_{i q}$ ses parties réelle et imaginaire, nous voyons d'abord que quand le point $M$ franchit la face $\varphi_{q}$ pour sortir de $\Pi$, la fonction $\Phi$ subit un saut brusque égal à

$$
\log \left|P\left(v_{i}-a_{i q}\right)\right|-\log \left|P\left(v_{i}\right)\right|=U_{q}\left(v_{i}\right) \text {. }
$$

Lorsque le point $\boldsymbol{M}$ franchit la transformée de la face $\varphi_{q}$ par l'addition de la période $a_{i}^{\prime}$, la fonction $\Phi$ subit un saut brusque égal à

$$
\log \left|P\left(v_{i}-a_{i}^{\prime}-a_{i q}\right)\right|-\log \left|P\left(v_{i}-a_{i}^{\prime}\right)\right|=U_{q}\left(v_{i}-a_{i}^{\prime}\right) .
$$

Considérons maintenant la fonction

$$
\Omega=\Phi+\frac{1}{\alpha}\left(J_{1}+J_{2}+\ldots+J_{2 p}\right) .
$$

Nous voyons tout de suite que cette fonction est harmonique sauf en deux sortes de points:

$I^{\circ}$ Ceux où $F$ s'annule; en ces points ce n'est pas $\Omega$, mais

$$
\Omega-\log \left|P\left(v_{i}\right)\right| \quad \text { ou } \quad \Omega-\log \left|P\left(v_{i}-a_{i}^{\prime}\right)\right|
$$

qui est harmonique. 
$2^{\circ}$ Ceux qui sont sur la frontière de deux ou plusieurs prismatoïdes, points pour lesquels les fonctions $\Phi$ et $J$ subissent une discontinuité analytique.

Parmi ces points nous distinguerons $I^{\circ}$ ceux qui n'appartiennent qu'à 2 prismatoïdes et qui forment des variétés à $2 p-\mathrm{I}$ dimensions, variétés qui ne sont autre chose que les faces $\varphi_{q}$ et leurs transformés; et $2^{\circ}$ ceux qui appartiennent à plus de 2 prismatoïdes et qui forment des variétés à $2 p-2$ dimensions ou à moins de $2 p-2$ dimensions.

En ce qui concerne les premiers, on voit tout de suite que la fonction $Q$ reste analytique, et en effet $\Phi$ subit un saut brusque égal à $U_{q}, J_{q}$ un saut brusque égal à $-\alpha U_{q}$; de sorte que le saut est nul pour $\Phi-\frac{1}{\alpha} J_{q}$ et par conséquent pour $Q$, et que $\Omega$ coïncide avec son prolongement analytique.

Il est aisé d'en conclure qu'il en est encore de même en ce qui concerne les autres points qui font partie de plus de 2 prismatoïdes.

Soit en effet $A$ l'un de ces points; il appartiendra également à plusieurs de faces $\varphi_{q}$ ou de leurs transformées. Reprenons l'intégrale

$$
J=\int\left(W \frac{d U^{\prime}}{d n}-\frac{d W}{d n} U^{\prime}\right) d \omega^{\prime}
$$

étendue à la face $\varphi$. D'après l'hypothèse, le point $A$ appartient à plusieurs des transformées de $\varphi$, par exemple aux transformées de $\varphi$ par l'addition des périodes $a_{i}^{(1)}, a_{i}^{(2)}, \ldots, a_{i}^{(m)}$, transformées que je désignerai par $\varphi^{(1)}, \varphi^{(2)}$, $\ldots, \varphi^{(m)}$. Si je représente alors par $A^{(1)}, A^{(2)}, \ldots, A^{(m)}$ les points qui se déduisent de $A$ par la soustraction des périodes $a_{i}^{(1)}, a_{i}^{(2)}, \ldots, a_{i}^{(n)}$, ces $m$ points seront tous sur $\varphi$, ou plutôt sur la frontière de la face $\varphi$.

Quand le point $M$ viendra en $A$ et le point $M^{\prime}$ en $A^{(k)}$, celle des expressions $\rho^{2-2 p}$ qui correspond à la période $a_{i}^{(k)}$ et que je représenterai par $\rho_{k}^{2-2 p}$ deviendra infinie. Nous avons donc $m$ de ces expressions à savoir

$$
\rho_{1}^{2-2 p}, \rho_{2}^{2-2 p}, \ldots, \rho_{m}^{2-2 p}
$$

qui peuvent devenir infinies quand $M$ est en $A$ et que $M^{\prime}$ est sur la face $\varphi$ ou sur la frontière de cette face. Toutes les autres expressions $\rho^{2-2 p}$ restent finies. 
Posons alors:

$$
\begin{aligned}
& \rho_{1}^{2-2 p}+\rho_{2}^{2-2 p}+\ldots+\rho_{m}^{2-2 p}=w \\
& W=W^{*}+w ; \quad J=J^{*}+j \\
& J^{*}=\int\left(W^{*} \frac{d U^{\prime}}{d n}-\frac{d W^{*}}{d n} U^{\prime}\right) d \omega^{\prime} \\
& j=\int\left(w \frac{d U^{\prime}}{d n}-\frac{d w}{d n} U^{\prime}\right) d \omega^{\prime}
\end{aligned}
$$

Comme la fonction $W^{*}$ reste régulière, quelle que soit la position de $M^{\prime}$ sur $\varphi$, quand le point $M$ est voisin de $A$, nous devons conclure que l'intégrale $J^{*}$ est régulière dans le voisinage de $A$.

Quant à $j$, elle est égale à l'intégrale

$$
\int\left(r^{2-2 r} \frac{d U^{\prime}}{d u}-\frac{d r^{2-2 p}}{d n} U^{\prime}\right) d \omega^{\prime}
$$

étendue, non plus à $\varphi$, mais à l'ensemble des transformées $\varphi^{(1)}, \varphi^{(2)}, \ldots, \varphi^{(m)}$. Soit $E$ cet ensemble. Il est clair que le point $A$ appartient à la variété $E$ et qu'il n'est pas sur la frontière de $E$ (car dans ce dernier cas, il $\mathrm{y}$ aurait de l'autre côté de cette frontière une transformée de $\varphi$ dont $A$ devrait faire partie et d'après notre hypothèse $\varphi^{(1)}, \varphi^{(2)}, \ldots, \varphi^{(m)}$ sont toutes les transformées de $\varphi$ dont $A$ fait partie).

L'intégrale (4) est une fonction holomorphe des $x$ et des $y$, sauf quand le point $M$ est sur $E$. Quand le point $M$ vient en $A$, la fonction $j$ éprouve donc une discontinuité analytique. Soit maintenant $E^{\prime}$ une autre variété à $2 p$ - I dimensions, ayant même frontière que $E$, de telle façon que $E$ et $E^{\prime}$ limitent un domaine $o$. Comme $A$ n'appartient pas à la frontière de $E$, nous pouvons supposer que $A$ ne fait pas partie de $E^{\prime}$.

Soit $j^{\prime}$ l'intégrale analogue à l'intégrale (4) étendue à $E^{\prime}$. Ce sera une fonction holomorphe des $x$ et des $y$ sauf quand $M$ est sur $E^{\prime}$. Elle sera donc holomorphe dans le voisinage du point $A$.

Or en vertu du théorème de Green, les deux intégrales $j$ et $j^{\prime}$ sont égales entre elles en dehors du domaine $o$. Donc l'intégrale $j$, et par conséquent aussi l'intégrale $J$, est susceptible d'être prolongée analytiquement au delà de la coupure dans le voisinage du point $A$.

C'est là ce que je voulais d'abord démontrer. 
Revenons maintenant à la fonction $\Omega$. D'après ce qui précède, cette fonction pourrait admettre pour coupures les diverses transformées des faces $\varphi_{q}$; mais elle est toujours susceptible d'être prolongée analytiquement au delà de ces coupures, même dans le voisinage de $A$. De plus nous avons vu que quand on franchit l'une de ces coupures, la fonction $\Omega$ coincide avec son prolongement analytique. La fonction $\Omega$ reste donc analytique et harmonique en $A$.

Il y aurait exception évidemment si au point $A$ la fonction $F$ s'annulait; ce serait alors $\Omega-\log \left|P\left(v_{i}\right)\right|$ ou $\Omega-\log \left|P\left(v_{i}-a_{i}^{\prime}\right)\right|$ qui serait analytique et harmonique.

La fonction $\Omega$ jouissant des mêmes propriétés que la fonction $\chi$ du paragraphe précédent et la fonction $V$ du tome 22, pages I 69 sqq, pourra. jouer le même rôle.

D'après la définition même de la fonction $W$, ses dérivées secondes sont des fonctions périodiques des $x$ et des $y$. Soit en effet $D W$ une des dérivées secondes de $W$ par rapport aux variables $x$ et $y$; plus généralement $D$ sera un signe de dérivation par rapport aux $x$ et aux $y$, et $D^{\prime}$ sera la dérivée correspondante prise par rapport aux variables $x^{\prime}$ et $y^{\prime}$.

Si alors $D$ est une dérivée seconde, nous aurons

$$
D W=\sum D_{\tau}, \quad D \rho^{2-2 p}=D \sigma+D \tau .
$$

Comme $\sigma$ est un polynôme du $2^{\mathrm{d}}$ degré, $D \sigma$ se réduit à une constante; d'autre part comme $\rho$ est une fonction des différences $x-x^{\prime}, y-y^{\prime}$, nous aurons:

$$
D \rho^{2-2 p}=D^{\prime} \rho^{2-2 p} .
$$

Comme $\tau$ ne contient que des termes du $3^{\text {e }}$ degré au moins, $D \tau$ est du $\mathrm{I}^{\text {er }}$ degré au moins et s'annule avec les $x$ et les $y$; nous avons:

$$
D^{\prime} \rho^{2-2 p}=D \sigma+D \tau \text {. }
$$

Soit $\rho_{0}$ ce que devient $\rho$ quand les $x$ et les $y$ s'annulent. La formule précédente devient donc pour $x=y=0$ :

$$
D^{\prime} \rho_{0}^{2-2 p}=D \sigma .
$$

Comme nous savons que $D \sigma$ est une constante, cette formule nous donne la valeur de $D_{\sigma}$ et nous pouvons écrire:

$$
D_{\tau}=D^{\prime} \rho^{2-2 p}-D^{\prime} \rho_{0}^{2-2 p} .
$$


La série

$$
D W=\sum\left(D^{\prime} \rho^{2-2 p}-D^{\prime} \rho_{0}^{2-2 p}\right)
$$

est absolument convergente. Quand $x$ et $y$ augmentent d'une période l'expression $\rho^{2-2 p}$ se transforme en une autre expression $\rho^{\prime 2-2 p}$. Quand $x^{\prime}$ et $y^{\prime}$ diminuent de la même période $\rho^{2-2 p}$ se change encore en $\rho^{2-2 p}$, mais en même temps $\rho_{0}^{2-2 p}$ se change en $\rho_{0}^{\prime 2-2 p}$. Nous pouvons écrire:

$$
D W=\sum\left(D^{\prime} \rho^{\prime 2-2 p}-D^{\prime} \rho_{0}^{\prime 2-2 p}\right)
$$

car les deux séries ne diffèrent que par l'ordre des termes.

$\mathrm{Si}$ maintenant $x$ et $y$ augmentent de la période en question, alors $D W$ devient

$$
\sum\left(D^{\prime} \rho^{\prime 2-2 p}-D^{\prime} \rho_{0}^{2-2 p}\right)
$$

donc $D W$ a augmenté de

$$
\sum\left(D^{\prime} \rho_{0}^{\prime 2-2 p}-D^{\prime} \rho_{0}^{2-2 p}\right) .
$$

Cette série est absolument convergente, et il est aisé de vérifier que la somme en est nulle. Donc $D W$ est une fonction périodique.

\section{Q. F. D}

Si $D W$ est périodique, il en sera de même de

$$
D J=\int\left(D W \frac{d U^{\prime}}{d n}-U^{\prime} \frac{d D W}{d n}\right) d \omega^{\prime} .
$$

D'ailleurs $D \Phi$ est périodique par définition et il doit en être de même de

$$
D \Omega=D \Phi+\frac{\mathrm{I}}{\alpha} \Sigma D J_{q} .
$$

Ainsi les dérivées secondes de $D \Omega$ sont des fonctions périodiques des $x$ et des $y$.

Nous allons introduire une autre fonction analogue à $\Omega$ et que jappellerai $\Omega^{\prime}$. A cet effet je commence par définir une intégrale analogue à $J$ et que j'appelle

$$
J^{\prime}=\int\left(W \frac{d V^{\prime}}{d n}-\frac{d W}{d n} V^{\prime}\right) d \omega^{\prime}
$$

Cette intégrale diffère de $J$ parce que $U^{\prime}$ y est remplacé par $V^{\prime} ; V^{\prime}$ est 
la valeur de la fonction $V$ au point $M^{\prime}$, et je rappelle que $V$ est la partie imaginaire de la fonction

$$
\log P\left(v_{i}-a_{i}\right)-\log P\left(v_{i}\right)
$$

dont $U$ est la partie réelle.

On voit que dans le voisinage du champ d'intégration envisagé, la fonction $V$ reste holomorphe.

Je définirai la fonction $\Phi^{\prime}$ comme il suit; dans le prismatoïde $\Pi^{\prime}$ (vide supra) elle sera égale à

$$
\text { arg. } P\left(v_{i}-a_{i}^{\prime}\right) \text {. }
$$

Elle ne sera donc définie qu'à un multiple près de $2 \pi$.

Je poserai

$$
\Omega^{\prime}=\Phi^{\prime}+\frac{1}{\alpha} \Sigma J_{g}^{\prime}
$$

et il est clair que la fonction $Q^{\prime}$ jouira des mêmes propriétés que la fonction $\Omega$, à cette différence près qu'elle ne sera déterminée qu'à un multiple près de $2 \pi$.

Quelles relations aurons-nous maintenant entre $\Omega$ et $\Omega^{\prime}$; la fonction $J$ étant analytique et harmonique sauf sur les coupures, et ayant pour prolongement analytique:

$$
J-\alpha U\left(x_{k}-b_{k}^{\prime}\right)
$$

la dérivée $D J$ sera analytique et harmonique sauf sur les coupures et aura pour prolongement analytique

$$
D J-\alpha D U\left(x_{k}-b_{k}^{\prime}\right) .
$$

De même la dérivée $D J^{\prime}$ sera analytique et harmonique sauf sur les coupures et aura pour prolongement analytique

$$
D J^{\prime}-a D V\left(x_{k}-b_{k}^{\prime}\right)
$$

Mais $U$ et $V$ sont les parties réelle et imaginaire d'une même fonction analytique des variables complexes $v$. On en conclut que chacune des dérivées secondes de $U$ est égale à une des dérivées secondes de $V$. On aura par exemple

$$
D U=D_{1} V
$$


Sur les fonctions abéliennes.

Comparons maintenant $D J$ et $D_{1} J^{\prime}$. Toutes deux sont harmoniques et analytiques sauf sur les coupures et elles ont respectivement pour prolongement analytique

$$
D J-\alpha D U\left(x_{k}-b_{k}^{\prime}\right), D_{1} J^{\prime}-\alpha D_{1} V\left(x_{k}-b_{k}^{\prime}\right)=D_{1} J^{\prime}-\alpha D U\left(x_{k}-b_{k}^{\prime}\right) .
$$

Ainsi la différence $D J-D_{1} J^{\prime}$ reste analytiquement continue sur les coupures; elle est donc analytique et harmonique dans tout l'espace.

De plus $D J$ de même que $D_{1} J^{\prime}$ est périodique; notre différence est donc analytique, harmonique et périodique dans tout l'espace; c'est dire qu'elle se réduit à une constante. (Cf AppeLL, Acta, tome 3.)

Il résulte de là que les dérivées de $D J$ sont égales aux dérivées correspondantes de $D_{1} J^{\prime}$

$$
\frac{d}{d x_{k}} D J=\frac{d}{d x_{k}} D_{1} J^{\prime} ; \quad \frac{d}{d y_{k}} D J=\frac{d}{d y_{k}} D_{1} J^{\prime}
$$

Quelle est maintenant la signification des équations

$$
D H=D_{1} H^{\prime},
$$

$H$ et $H^{\prime}$ désignant deux fonctions quelconques des $x$ et des $y$. Pour nous en rendre compte formons explicitement nos équations:

$$
D U=D_{1} V
$$

Nous avons:

d'où:

$$
\frac{d U}{d x_{k}}=\frac{d V}{d y_{k}}, \quad \frac{d U}{d y_{k}}=-\frac{d V}{d x_{k}}
$$

$\frac{d^{2} U}{d x_{k} d x_{j}}=\frac{d^{2} V}{d y_{k} d x_{j}}, \quad \frac{d^{2} U}{d x_{k} d !_{j}}=\frac{d^{2} V}{d y_{k} d y_{j}}, \quad \frac{d^{2} U}{d y_{k}} \frac{d x_{j}}{d x_{k}}=-\frac{d^{2} V}{d x_{j}}, \quad \frac{d^{2} U}{d y_{k} d y_{j}}=-\frac{d^{2} V}{d x_{k} d y_{j}}$.

Ce sont là nos équations (6). Pour avoir les équations (5), il suffit de remplacer $U$ et $V$ par $H$ et $H^{\prime}$. Ces équations peuvent donc s'écrire:

$$
\frac{d}{d x_{k}} \frac{d H}{d x_{j}}=\frac{d}{d y_{k}} \frac{d H^{\prime}}{d x_{j}}, \quad \frac{d}{d y_{k}} \frac{d H}{d x_{j}}=-\frac{d}{d x_{k}} \frac{d H^{\prime}}{d x_{j}}
$$

avec les équations qu'on en déduirait en remplaçant $x_{j}$ par $y_{j}$. 
Ces équations signifient que

$$
\frac{d H}{d x_{j}}+\sqrt{-\mathrm{I}} \frac{d H^{\prime}}{d x_{j}}, \quad \frac{d H}{d y_{j}}+\sqrt{-\mathrm{I}} \frac{d H^{\prime}}{d y_{j}},
$$

c'est à dire les dérivées premières de $H+\sqrt{-1} H^{\prime}$, sont des fonctions analytiques des variables complexes $v$.

Nos équations (4) qui peuvent s'écrire:

$$
D \frac{d J}{d x_{k}}=D_{1} \frac{d J^{\prime}}{d x_{k}}, \quad D \frac{d J}{d y_{k}}=D_{1} \frac{d J^{\prime}}{d y_{k}}
$$

signifient alors que les dérivées secondes de $J+\sqrt{-1} J$ ' sont des fonctions analytiques des variables complexes $v$.

Donc $J+\sqrt{-1} J^{\prime}$ sera égal à une fonction analytique des $v$, plus un polynôme du second degré par rapport aux $x$ et aux $y$.

Comme $\Phi+\sqrt{-1} \Phi^{\prime}$ est par définition une fonction analytique de $v$, nous devons conclure que $\Omega+\sqrt{-1} Q^{\prime}$ est égal à une fonction analytique des $v$, plus un polynôme du $2^{\mathrm{d}}$ degré par rapport aux $x$ et aux $y$; toutefois cette fonction devient logarithmiquement infinie aux points où $F$ s'annule.

Soit $\bar{\omega}$ le polynôme du $2^{\text {d }}$ degré dont il vient d'être question; alors:

$$
\Omega+\sqrt{-\mathrm{I}} \Omega^{\prime}-\overline{\boldsymbol{\omega}} \quad \text { et } \quad \boldsymbol{\theta}=e^{\Omega+\sqrt{-1} g-\tilde{\omega}}
$$

sont des fonctions analytiques des variables complexes $v$; la dernière $\boldsymbol{\theta}$ est une fonction entière; en effet elle ne pourrait cesser d'être holomorphe qu'aux points où $F$ s'annule, en ces points

$$
\Omega+\sqrt{-\mathrm{I}} \Omega^{\prime}-\overline{\boldsymbol{\omega}}-\log P\left(v_{i}-a_{i}^{\prime}\right)=\Lambda
$$

est holomorphe et par conséquent

est holomorphe également.

$$
\theta=P\left(v_{i}-a_{i}^{\prime}\right) e^{i}
$$

I Les dérivées secondes de $\Omega+\sqrt{-\mathrm{I}} \Omega^{\prime}-\bar{\omega}$ sont périodiques; nous l'avons vu pour $Q$; nous le verrions de la même manière pour $Q^{\prime}$, et les dérivées secondes de $\bar{\omega}$ se réduisent à des constantes.

Il résulte immédiatement de là que $\theta$ se reproduit multiplié par une exponentielle dont l'exposant est un polynôme $d u I^{\text {er }}$ degré, quand les variables augmentent d'une période. 
C'est une sfonction intermédiaire" pour employer l'expression assez mal justifiée de Briot et Bovquet. Notre fonction $F$ est done le quotient de deux fonctions intermédiaires.

\section{§5. Des fonctions intermédiaires.}

L'étude des fonctions abéliennes se trouve ainsi naturellement ramenée à celle des s fonctions intermédiaires,, c'est à dire des fonctions entières qui se reproduisent par l'addition d'une période, à un multiplicateur près qui est une exponentielle du I $^{\text {er }}$ degré.

C'est principalement dans le tome 8 de l'American Journal of Mathematics (i 886 ), que se trouvent exposées mes recherches sur ces fonctions intermédiaires.

On reconnaît tout de suite que les périodes et les multiplicateurs ne peuvent pas être quelconques.

Supposons que quand $v_{i}^{\prime}$, augmentant d'une période, se change en $v_{i}^{\prime}+a_{i k}$, le logarithme de la fonction intermédiaire augmente de

$$
P_{k}=\Sigma a_{i k} v_{i}+\beta_{k}
$$

de telle façon que le multiplicateur correspondant à la période $a_{i k}$ soit $e^{P_{k}}$; on voit que le nombre:

$$
M_{k j}=\sum_{i=1}^{p}\left(\alpha_{i j} a_{i k}-\alpha_{i k} a_{i j}\right)
$$

doit être égal à un entier multiplié par $2 \pi \sqrt{-\mathrm{r}}$. Il suffit pour s'en rendre compte d'exprimer que l'on obtient le même résultat en ajoutant d'abord la période $a_{i k}$, puis la période $a_{i j}$, ou en ajoutant ces deux mêmes périodes dans l'ordre invers.

Ces nombres $M_{k j}$ et les relations précédentes ont été étudiées par M. Frobenius dans le tome 97 de Crelle dans son mémoire sur les fonctions Jacobiennes. J'ai repris cette étude à un autre point de vue dans le mémoire cité de l'American Journal.

Les fonctions intermédiaires se ramènent immédiatement aux fonctions $\theta$; c'est à dire à celles où tous les nombres (pour un choix convenable des périodes) $M_{k j}$ sont nuls, sauf $p$ d'entre eux qui sont d'ailleurs tous égaux entre eux. En général cette réduction ne peut pas se faire de 
H. Poincaré.

plusieurs manières essentiellement différentes; mais il n'en est pas de même quand les fonctions abéliennes considérées peuvent par une transformation convenable être ramenées soit aux fonctions elliptiques, soit à des fonctions abéliennes de rang moindre, e'est à dire dans les cas de réduction dont je parlerai plus loin.

Depuis M. Humbert a reconnu qu'il existe d'autres systèmes de périodes pour lesquelles, bien qu'on ne soit pas dans un des cas de réduction, il existe des fonctions intermédiaires singulières réductibles de plusieurs manières aux fonctions $\theta$. Les fonctions de M. Humber'T ont une grande importancè.

Il est aisé de voir quelle est la signification de ces nombres $\boldsymbol{M}_{k j}$.

Prenons le prismatoïde des périodes $\Pi$; c'est un domaine de l'espace à $2 p$ dimensions. A chacune des

$$
\frac{\mid(2 p)}{|\underline{q}| \underline{(2 p-q)}}
$$

combinaisons des $2 p$ périodes $q$ à $q$, correspond un système de polyèdres en forme de "parallélotopes» ou de "prismatoïdes" de l'espace à $q$ dimensions, qui sont tous parallèles et égaux entre elles et qui sont au nombre de:

$$
2^{2 p-q} \text {. }
$$

C'est ainsi que dans l'espace ordinaire $(2 p=3)$, un parallélépipède a six faces $(q=2)$ parallèles deux à deux $\left(2^{2 p-q}=2\right)$ et réparties par conséquent

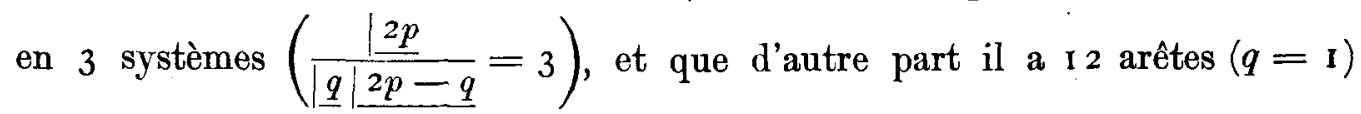
parallèles quatre à quatre $\left(2^{2 p-q}=4\right)$ et réparties par conséquent en 3 systèmes $\left(\frac{\underline{2 p}}{\underline{q} \underline{2 p-q}}=3\right)$.

Parmi ces variétés nous avons considéré dans les paragraphes précédents celles qui correspondent à $q=2 p-1$ et que nous avons appelées les faces $\mathrm{du}$ prismatoïde. Nous envisagerons maintenant plus particulièrement celles qui correspondent à $q=\mathrm{I}$ et que nous appellerons les arêtes, et celles qui correspondent à $q=2$ et que nous appellerons, non pas les faces, mais les parallèlogrammes (en abrégé prlg) du prismatoïde.

A chaque arête correspond donc une période, à chaque prlg un des nombres $M_{k j}$. 
Soit alors $\theta$ une fonction intermédiaire quelconque et considérons l'intégrale

$$
\int d \log \theta
$$

que nous prendrons le long du périmètre de l'un de nos prlg., correspondant par exemple au nombre $\boldsymbol{M}_{k j}$.

Le long $\mathrm{du} \mathrm{I}^{\mathrm{er}}$ côté, l'intégrale sera:

$$
\Sigma \alpha_{i k} v_{i}^{0}+\beta_{k}
$$

si les coordonnées $\mathrm{du} \mathrm{I}^{\text {er }}$ sommet sont $v_{i}^{0}$; le long du $2^{\mathrm{d}}$ côté, elle sera

$$
\Sigma \alpha_{i j}\left(v_{i}^{0}+a_{i k}\right)+\beta_{j}
$$

le long du $3^{\mathrm{e}}$

$$
-\Sigma \alpha_{i k}\left(v_{i}^{0}+a_{i j}\right)-\beta_{k}
$$

et enfin le long du $4^{\mathrm{e}}$ :

$$
-\Sigma \alpha_{i j} v_{i}^{0}-\beta_{j}
$$

L'intégrale totale est donc

$$
\Sigma\left(\alpha_{i j} a_{i k}-\alpha_{i k} a_{i j}\right)=\boldsymbol{M}_{k j} .
$$

Nous pouvons décomposer la surface de notre prlg en une infinité de contours plans fermés infiniment petits; la somme des intégrales

$$
\int d \log \theta
$$

prises le long de ces différents contours sera égale à $\boldsymbol{M}_{k j}$. Comme la quantité sous le signe $\int$ est une différentielle exacte, l'intégrale prise le long de l'un de ces contours sera nulle à moins que la fonction sous le signe $\int$ ne devienne infinie à l'intérieur du contour, c'est à dire que $\theta$ ne s'annule à l'intérieur du contour.

Nous sommes donc conduits à rechercher les zéros de $\boldsymbol{\theta}$ situés sur la surface des prlg; c'est à dire les intersections de la variété à $2 p-2$ dimensions $\boldsymbol{\theta}=\mathrm{O}$ avec le plan du prlg.

Je supposerai que nous n'avons que des intersections simples, c'est à dire que ce plan et la variété $\theta=0$ ne sont pas tangents l'un à l'autre. Il est clair que sauf des cas exceptionnels, il suffira de déplacer d'une façon convenable le prismatoïde parallèlement à lui-même pour qu'un pareil 
contact n'ait pas lieu. D'ailleurs dans ces cas exceptionnels, où ce contact ne peut-être évité, les résultats que je vais démontrer s'appliquent encore, grâce à une généralisation presque immédiate.

Quelle est la condition pour que ce contact ait lieu? Soient $\theta_{0}$ et $\theta_{1}$ les parties réelle et imaginaire de $\theta, x_{i}$ et $y_{i}$ celles de $v_{i}$; alors $\theta_{0}$ et $\theta_{1}$ sont des fonctions des $x$ et des $y$; soient $b_{i k}$ et $c_{i k}$ celles de $a_{i k}$.

La condition cherchée peut s'écrire:

$$
\begin{aligned}
& D_{k j}=\sum_{i}\left(b_{i k} \frac{d \theta_{0}}{d x_{i}}+c_{i k} \frac{d \theta_{0}}{d y_{i}}\right) \sum_{i}\left(b_{i j} \frac{d \theta_{1}}{d x_{i}}+c_{i j} \frac{d \theta_{1}}{d y_{i}}\right) \\
& -\sum_{i}\left(b_{i j} \frac{d \theta_{0}}{d x_{i}}+c_{i k} \frac{d \theta_{0}}{d y_{i}}\right) \sum_{i}\left(b_{i k} \frac{d \theta_{1}}{d x_{i}}+c_{i k} \frac{d \theta_{i}}{d y_{i}}\right)=0 .
\end{aligned}
$$

Nous supposerons donc que l'expression $D$ n'est pas nulle en même temps que $\theta$ à l'intérieur du prlg. Considérons donc un des zéros de $\theta$ à l'intérieur de notre prlg. Notre intégrale prise le long d'un contour entourant ce zéro sera évidemment égale à

$$
\pm 2 \pi \sqrt{-\mathrm{I}} \text {. }
$$

Quant au signe il dépendra évidemment de celui de $D_{k j}$. Nous pouvons faire des conventions telles que ce signe soit celui de $D_{k j}$.

Nous sommes ainsi amenés à distinguer les zéros pour lesquels $D_{k j}$ est positif de ceux pour lesquels $D_{k j}$ est négatif; je les appellerai pour abréger les zéros positifs et les zéros négatifs; et j'arrire à cette conclusion que le nombre entier

$$
\frac{M_{k j}}{2 \pi \sqrt{-1}}
$$

est égal à l'excès du nombre des zéros positifs sur celui des zéros négatifs. Quand on permute les indices $k$ et $j$, le nombre $M_{k j}$ change de signe; il en est de même de $D_{k j}$; le prlg reste le même, mais il est parcouru en sens contraire.

Ce résultat est le même que celui que javais obtenu par une tout autre voie dans le tome 9 des Acta mathematica, pages 369 sqq.

Considérons un segment de droite quelconque dont la projection sur l'axe des $x_{i}$ soit $\xi_{i}$ et dont la projection sur l'axe des $y_{i}$ soit $\eta_{i}$. Posons

$$
\xi_{i}=\sum_{k=1}^{2 p} \mu_{k} b_{i k} ; \quad \eta_{i}=\sum_{k=1}^{2 p} \mu_{k} c_{i k}
$$


les coëfficients $\mu$ ainsi définis pourront s'appeler les coëfficients caractéristiques du segment considéré.

La longueur du segment est la racine carrée de

$$
\sum \xi^{2}+\sum \eta^{2}=\phi(\mu)
$$

$\psi(\mu)$ étant une forme quadratique définie positive par rapport à $\mu_{1}, \mu_{2}, \ldots, \mu_{2 p}$.

Lorsque les $\mu$ sont entiers, le segment représente en grandeur, direction et sens une période, $\Sigma \mu_{k} a_{i k}$, et les quantités a correspondantes seront $\sum \mu_{k} \alpha_{i k}$.

Cela posé, envisageons deux segments ayant respectivement pour coefficients caractéristiques $\mu_{k}$ et $\mu_{k}^{\prime}$, et pour projections sur les axes $\xi_{i}, \eta_{i}$ et $\xi_{i}^{\prime}, \eta_{i}^{\prime}$. La surface du parallélogramme construit sur ces deux segments sera la racine carrée de:

$$
\Omega\left(\mu, \mu^{\prime}\right)=\phi(\mu) \phi\left(\mu^{\prime}\right)-\frac{\mathrm{I}}{4}\left(\sum \mu_{k}^{\prime} \frac{d \psi}{d \mu_{k}}\right)^{2}
$$

Si les $\mu$ et les $\mu^{\prime}$ sont des entiers, et que les deux segments soient des périodes, nous pouvons nous proposer de calculer le nombre $M$ correspondant; pour cela dans l'expression de $M_{k j}$. il faut remplacer

$$
a_{i k}, \alpha_{i k}, a_{i j}, \alpha_{i j}
$$

par

$$
\Sigma \mu_{k} a_{i k}, \Sigma \mu_{k} \alpha_{i k}, \Sigma \mu_{i}^{\prime} a_{i k}, \Sigma \mu_{i}^{\prime} \alpha_{i k}
$$

On trouve ainsi:

$$
M\left(\mu, \mu^{\prime}\right)=\Sigma M_{k j}\left(\mu_{k} \mu_{j}^{\prime}-\mu_{k}^{\prime} \mu_{j}\right)
$$

la sommation étant étendue à toutes les combinaisons des deux indices $k$ et $j$. On voit que $M$ est une forme bilinéaire par rapport aux coefficients $\mu$ et $\mu^{\prime}$.

De même pour calculer le nombre $D$ correspondant à notre parallélogramme il faut dans l'expression de $D_{k j}$ remplacer $b_{i k}, c_{i k}, b_{i j}, c_{i j}$ par

$$
\Sigma \mu_{k} b_{i k}, \Sigma \mu_{k} c_{i k}, \Sigma \mu_{k}^{\prime} b_{i k}, \Sigma \mu_{k}^{\prime} c_{i k},
$$

ce qui donne:

$$
D\left(\mu, \mu^{\prime}\right)=\Sigma D_{k j}\left(\mu_{k} \mu_{j}^{\prime}-\mu_{k}^{\prime} \mu_{j}\right)
$$

Si nous distinguons encore les zéros positifs ou négatifs suivant le signe 
de $D\left(\mu, \mu^{\prime}\right)$, nous verrons que l'excès du nombre des zéros positifs sur le nombre des zéros négatifs, est sur notre parallélogramme:

$$
\frac{M\left(\mu, \mu^{\prime}\right)}{2 \pi \sqrt{-1}}
$$

Considérons maintenant une aire quelconque dans le plan de ce parallèlogramme qui soit limitée par des segments représentant des périodes en grandeur et direction. Cette aire sera décomposable en un certain nombre $n$ de parallélogrammes égaux, et on aura:

$S$ étant la surface de l'aire.

$$
n=\frac{S}{\sqrt{\bar{\Omega}}} \frac{S}{\left(\mu, \mu^{\prime}\right)}
$$

L'excès du nombre des zéros positifs sur les zéros négatifs sera:

$$
n \frac{M\left(\mu, \mu^{\prime}\right)}{2 \pi \sqrt{-1}}=\frac{S}{2 \pi \sqrt{-1}} \frac{M\left(\mu, \mu^{\prime}\right)}{\sqrt{\Omega\left(\mu, \mu^{\prime}\right)}} .
$$

On remarquera que le rapport

$$
\frac{M\left(\mu, \mu^{\prime}\right)}{\left.\sqrt{\overline{\Omega(\mu}}, \mu^{\prime}\right)}
$$

ne change pas, ni quand on multiplie tous les $\mu$ par un même facteur constant, ni quand on multiplie tous les $\mu^{\prime}$ par un même facteur constant. Il ne change pas non plus quand on fait subir à $\mu_{1}$ et à $\mu_{1}^{\prime}$ une substitution linéaire, et en même temps à $\mu_{k}$ et $\mu_{k}^{\prime}$ la même substitution linéaire.

Ce rapport dépend done seulement au moins en valeur absolue de la direction du plan du parallélogramme; cependant il changerait de signe si la substitution linéaire dont je viens de parler avait un déterminant négatif.

Considérons maintenant une aire qui ne soit plus plane, mais qui soit limitée par des sègments de droite représentant en grandeur et direction des périodes.

Considérons les zéros de $\boldsymbol{\theta}$ qui sont sur cette aire; nous distinguerons les zéros positifs et négatifs d'après le signe de $D\left(\mu, \mu^{\prime}\right)$, en appelant $\mu_{k}$ et $\mu_{k}^{\prime}$ les coefficients caractéristiques de deux droites menées dans le plan tangent à l'aire au point envisagé. Nous remarquerons que $D\left(\mu, \mu^{\prime}\right)$ ne change pas quand on fait subir à $\mu_{1}$ et ̀̀ $\mu_{1}^{\prime}$ une substitution linéaire de déterminant $+\mathrm{I}$, et en même temps à $\mu_{k}$ et $\mu_{k}^{\prime}$ la même substitution linéaire. 
Je me propose alors de démontrer que l'excès $N$ du nombre des zéros positifs sur celui des zéros négatifs est égal à

$$
N=\int \frac{d \sigma}{2 \pi \sqrt{-\mathrm{I}}} \frac{M\left(\mu, \mu^{\prime}\right)}{\sqrt{\Omega\left(\mu, \mu^{\prime}\right)}}
$$

$d \sigma$ représentant un élément de l'aire, et $\mu$ et $\mu^{\prime}$ les coefficients caractéristiques de deux droites menëes dans le plan tangent.

Je diviserai la démonstration en trois parties:

$I^{\circ} \mathrm{Je}$ suppose d'abord que l'aire se décompose en un certain nombre de parallélogrammes construits sur des périodes; la formule est alors une conséquence immédiate de celle que nous venons d'établir.

$2^{\circ} \mathrm{Je}$ suppose que l'aire soit fermée.

Dans ce cas je remarque d'abord que $N$ est nul. En effet l'aire étant fermée peut être considérée comme la frontière complète d'une variété $V$ à 3 dimensions. Les points de cette variété où $\theta=0$ formeront une ligne; les points où cette ligne percera la frontière pour entrer dans $V$ seront les zéros positifs, ceux où elle percera la frontière pour sortir de $V$ seront les zéros négatifs. Il en résulte immédiatement que le nombre des zéros des 2 espèces est le même.

D'autre part, je dis que l'intégrale double du second membre est nulle; on peut l'écrire en effet:

$$
\sum \frac{M_{k j}}{2 \pi \sqrt{-\mathrm{I}}} \int d \mu_{k}^{0} d \mu_{j}^{0}
$$

en désignant par $\mu_{k}^{0}$ les coefficients caractéristiques du vecteur qui va de l'origine au centre de gravité de l'élément $d \sigma$.

L'aire étant fermée, on voit aisément que chacune des intégrales $\int d \mu_{k}^{0} d \mu_{j}^{0}$ est nulle.

$3^{\circ} \mathrm{Je}$ suppose enfin que l'aire $A$ soit quelconque, mais limitée par des segments représentant des périodes.

Dans ce cas je puis construire une aire $A^{\prime}$ ayant même frontière que $A$ et décomposable en parallélogrammes construits sur des périodes. Le théorème est vrai pour $A^{\prime}$, il est vrai pour l'aire totale $A+A^{\prime}$ qui est fermée; il est done vrai pour $A$.

Supposons enfin que l'aire $A$ soit tout à fait quelconque et ne soit plus limitée par des segments représentant des périodes. Dans quelle mesure 
l'équation (2) restera-t-elle vraie? Supposons que l'aire $A$ soit très-grande, que par exemple ses dimensions linéaires soient des infinis grands du $I^{\mathrm{e}}$ ordre et sa surface un infini $d u 2^{d}$ ordre. Je dis alors que l'égalité (2) dont les deux membres sont des infinis grands du $2^{\mathrm{d}}$ ordre, reste vraie à des quantités près infiniment grandes du ${ }^{{ }^{e r}}$ ordre.

Nous pouvons en effet construire une ligne brisée fermée, composée de segments représentant des périodes, et s'écartant peu du périmètre de l'aire $A$; je veux dire par là que la distance de cette ligne brisée à ce périmètre restera plus petite que la plus grande diagonale du prismatoide $I$. Nous pourrons alors construire une aire $A^{\prime}$, limitée d'une part par le périmètre de $A$, d'autre part par cette ligne brisée, et dont la surface sera du même ordre que le périmètre de $A$, c'est à dire du $\mathbf{I}^{\text {er }}$ ordre.

Soient alors $N$ et $J$ la valeur des deux membres de l'égalité (2), (c'est à dire de l'excès de l'intégrale double) pour l'aire $A$, soient $N^{\prime}$ et $J^{\prime}$ les deux quantités correspondantes pour l'aire $A^{\prime} ; N+N^{\prime}$ et $J+J^{\prime}$ les deux quantités correspondantes pour l'aire $A+A^{\prime}$.

Comme l'aire $A+A^{\prime}$ est limitée par des segments représentant des périodes, on a

$$
N+N^{\prime}=J+J^{\prime} .
$$

D'autre part $N^{\prime}$, et $J^{\prime}$ sont du même ordre que la surface de $A^{\prime}$ c'est à dire du ${ }^{\text {er }}$ ordre. Donc

$$
\text { aux quantités près du } \mathrm{I}^{\text {er }} \text { ordre. } \quad \text { C. Q. F. D. }
$$

Si en particulier l'aire $A$ est plane, mais quelconque, on aura aux quantités près du $\mathbf{I}^{\text {er }}$ ordre

$$
N=\frac{S}{2 \pi \sqrt{-\mathrm{I}}} \frac{M\left(\mu, \mu^{\prime}\right)}{\sqrt{\Omega\left(\mu, \mu^{\prime}\right)}} .
$$

Les $\mu_{k}$ et les $\mu_{k}^{\prime}$ sont les coefficients caractéristiques de deux droites quelconques du plan; nous n'avons plus besoin de supposer qu'ils sont entiers ou même commensurables.

Mais parmi les plans possibles, nous distinguerons ceux qui sont papallèles à un plan $P$ donné par les équations:

$$
\frac{v_{1}}{\beta_{1}}=\frac{v_{2}}{\beta_{2}}=\ldots=\frac{v_{p}}{\beta_{p}}
$$

les $\beta$ étant des coefficients complexes quelconques. 
Soient $\gamma_{i}$ et $\hat{o}_{i}$ les parties réelle et imaginaire de $\beta_{i}$, nous pourrons envisager en particulier deux droites du plan $P$ parallèles respectivement aux deux droites:

$$
\frac{x_{i}}{\gamma_{i}}=\frac{x_{k}}{\gamma_{k}}=\frac{y_{i}}{\delta_{i}}=\frac{y_{k}}{\delta_{k}}, \quad \frac{x_{i}}{-\delta_{i}}=\frac{x_{k}}{-\delta_{k}}=+\frac{y_{i}}{\gamma_{i}}=+\frac{y_{k}}{\delta_{k}}
$$

Ce sont les coefficients caractéristiques de ces deux droites que nous appellerons respectivement $\mu_{k}$ et $\mu_{k}^{\prime}$ de sorte que nous aurons:

$$
\left\{\begin{aligned}
r_{i} & =\Sigma b_{i k} \mu_{k}, \quad \hat{o}_{i}=\Sigma c_{i k} \mu_{k}, \\
-\delta_{i} & =\Sigma b_{i k} \mu_{k}^{\prime}, \quad+\gamma_{i}=\Sigma c_{i k} \mu_{k}^{\prime}
\end{aligned}\right.
$$

et par conséquent:

$$
\begin{gathered}
D=\sum\left(r_{i} \frac{d \theta_{0}}{d x_{i}}+\delta_{i} \frac{d \theta_{0}}{d y_{i}}\right) \sum\left(-\delta_{i} \frac{d \theta_{1}}{d x_{i}}+\gamma_{i} \frac{d \theta_{1}}{d y_{i}}\right) \\
-\sum\left(r_{i} \frac{d \theta_{1}}{d x_{i}}+\delta_{i} \frac{d \theta_{1}}{d y_{i}}\right) \sum\left(-\delta_{i} \frac{d \theta_{0}}{d x_{i}}+\gamma_{i} \frac{d \theta_{0}}{d y_{i}}\right) .
\end{gathered}
$$

Or il est aisé de voir que

$$
\sum \beta_{i} \frac{d \theta}{d v_{i}}
$$

a pour partie réelle:

$$
\sum\left(r_{i} \frac{d \theta_{0}}{d x_{i}}+\delta_{i} \frac{d \theta_{0}}{d y_{i}}\right)=\sum\left(-\delta_{i} \frac{d \theta_{1}}{d x_{i}}+r_{i} \frac{d \theta_{1}}{d y_{i}}\right)
$$

et pour partie imaginaire

$$
\sum\left(r_{i} \frac{d \theta_{1}}{d x_{i}}+\delta_{i} \frac{d \theta_{1}}{d y_{i}}\right)=-\sum\left(-\delta_{i} \frac{d \theta_{0}}{d x_{i}}+r_{i} \frac{d \theta_{0}}{d y_{i}}\right)
$$

de sorte que

est essentiellement positif.

$$
D=\left|\sum \beta_{i} \frac{d \theta}{d v_{i}}\right|^{2}
$$

Il n'y a plus alors que des zéros positifs.

Le rapport $\frac{N}{S}$ représente alors la densité moyenne des zéros dans un plan parallèle à $P$, c'est à dire la limite vers laquelle tend le rapport du Acta mathematica. 26. Imprimé le $2 \bar{c}$ avril 1902. 
nombre des zéros contenus dans une aire située dans ce plan, à la surface de cette aire, quand cette surface croit indéfiniment. Cette densité moyenne est donc égale à

$$
\frac{1}{2 \pi \sqrt{-1}} \frac{M\left(\mu, \mu^{\prime}\right)}{\sqrt{\Omega}}
$$

les coefficients $\mu$ et $\mu^{\prime}$ étant déterminées par les équations (4).

Il résulte de là que pour ces valeurs des $\mu$ et des $\mu^{\prime}$, l'expression

$$
\frac{\mathbf{I}}{2 \pi \sqrt{-1}} M\left(\mu, \mu^{\prime}\right)
$$

est essentiellement positive.

Ce résultat peut se mettre encore sous d'autres formes.

Posons

$$
\begin{array}{ll}
\xi_{i}=\Sigma b_{i k} \mu_{k}, & \xi_{i+p}=\Sigma c_{i k} \mu_{k}, \\
\xi_{i}^{\prime}=\Sigma b_{i k} \mu_{k}^{\prime}, & \xi_{i+p}^{\prime}=\Sigma c_{i k} \mu_{k}^{\prime},
\end{array}
$$

alors notre expression

$$
\frac{M\left(\mu, \mu^{\prime}\right)}{2 \pi \sqrt{-1}}
$$

deviendra une forme bilinéaire des $\xi$ et des $\xi^{\prime}$ que je puis écrire:

$$
\Sigma A_{k j}\left(\xi_{j} \xi_{k}^{\prime}-\xi_{k} \xi_{j}^{\prime}\right)
$$

Cette forme devra être essentiellement positive si l'on y fait:

$$
\xi_{i}=\gamma_{i}, \quad \xi_{i+p}=\delta_{i}, \quad \xi_{i}^{\prime}=-\grave{o}_{i}, \quad \xi_{i+f}^{\prime}=r_{i} .
$$

Or par cette substitution notre forme doit devenir une forme quadratique par rapport aux $\gamma$ et aux $o$; cette forme quadratique doit être définie positive.

Posons maintenant:

$$
\mu_{k}+\sqrt{-1} \mu_{k}^{\prime}=x_{k}, \quad \mu_{k}-\sqrt{-1} \mu_{k}^{\prime}=x_{k}^{0},
$$

les équations (4) nous donneront:

Il vient alors:

$$
\Sigma a_{i k} x_{k}=0, \quad \Sigma a_{i k} x_{k}^{0}=2 \beta_{i} .
$$

$$
\mu_{k} \mu_{j}^{\prime}-\mu_{j} \mu_{k}^{\prime}=\left(x_{k} x_{j}^{0}-x_{j} x_{k}^{0}\right) \frac{-\mathbf{I}}{2 \sqrt{-1}}
$$


de sorte que:

$$
\frac{M}{2 \pi \sqrt{-1}}=\frac{1}{4 \pi} \Sigma M_{k j}\left(x_{k} x_{j}^{0}-x_{j} x_{k}^{0}\right) .
$$

La forme $\dot{a}$ variables conjuguées qui figure dans le $2^{\mathrm{d}}$ membre est donc essentiellement positive, si $\Sigma a_{i k} x_{k}$ est assujetti $\dot{a}$ étre nul.

On reconnaît là l'inégalité de Riemann, généralisée par Frobenius (Crelle, tome 97, page 21). Mais la voie par laquelle nous y sommes parvenus diffère beaucoup à la fois de celle de Riemann et de celle de Frobenius.

Je crois que les considérations qui précèdent sont de nature à mieux faire comprendre la signification des nombres $M_{k j}$, et les relations de ces nombres avec la répartition des zéros des fonctions $\boldsymbol{\theta}$.

\section{6. Cas de réduction.}

Dans quels cas une intégrale abélienne appartenant à une courbe de genre $p$ peut-elle être réduite à une intégrale abélienne appartenant à une courbe de genre moindre?

Dans quels cas une courbe de genre $p$ admettra-t-elle $q$ intégrales abéliennes de $1^{\text {ère }}$ espèce, admettant seulement $2 q$ périodes? $\mathrm{Ou}$, ce qui revient au même, dans quels cas une intégrale abélienne appartenant à une courbe de genre $p$ peut-elle être calculée à l'aide de systèmes de fonctions abéliennes de rang moindre que $p$, c'est à dire admettant moins de $p$ variables?

Enfin dans quels cas des fonctions abéliennes de rang $p$ peuvent-elles être réduites à des fonctions abéliennes de rang moindre?

Telles sont les questions qui ont occupé d'abord Weicrstrass et M. PiCARD et que j'ai abordées à mon tour, d'abord dans le Bulletin de la Société Mathématique de France (tome I 2), puis dans le tome 8 de l'American Journal.

Nous trouvons d'abord le cas où la fonction $\boldsymbol{\theta}$ peut être regardée comme le produit de $p$ fonctions $\theta$ elliptiques. C'est ce que j'appelle le cas singulier elliptique.

Puis nous avons le cas où la fonction $\theta$ est le produit de plusieurs fonctions $\boldsymbol{\theta}$ abéliennes de rang moindre. C'est ce que j'appelle le cas singulier abélien. 
Nous avons ensuite les cas, qui par une transformation d'ordre convenable, peuvent être ramenés soit au cas singulier elliptique, soit au cas singulier abélien.

Il n'y a pas d'autres cas de réduction.

Mais, circonstance remarquable, un système quelconque de périodes, diffère toujours infiniment peu d'une infinité de systèmes correspondant à des cas de réduction, de la même façon qu'un nombre réel quelconque diffère toujours infiniment peu d'une infinité de nombres rationnels.

Enfin si un système de fonctions abéliennes peut de deux manières différentes être ramené par une transformation, au cas singulier elliptique, cette réduction peut se faire d'une infinité de manières différentes. C'est ce qu'avait déjà remarqué M. Picand dans certains cas particuliers.

Je me borne à énoncer succinctement ces résultats; mais je dois cependant expliquer le parti qu'on en peut tirer pour l'étude des fonctions abéliennes les plus générales.

On peut les utiliser de 3 manières différentes:

$1^{\circ}$ On peut résoudre un problème dans le cas singulier elliptique, et montrer ensuite que le résultat ne peut être différent dans ce cas singulier de ce qu'il est dans le cas général.

C'est ainsi par exemple que j'ai déterminé le nombre des zéros communs à $p$ fonctions $\theta$; et M. Wirtinger a également tiré un grand parti de ce procédé.

$2^{\circ}$ On peut profiter de cette circonstance signalée plus haut qu'étant donnée une fonction abélienne quelconque, on peut toujours trouver une infinité de fonctions abéliennes réductibles qui en diffèrent aussi peu qu'on le veut.

C'est comme cela que j'ai déterminé la somme des zéros communs à $p$ fonctions $\boldsymbol{\theta}$.

$3^{\circ}$ On peut étudier spécialement les cas qui diffèrent peu du cas singulier elliptique, ou du cas singulier abélien.

C'est ce que j'ai fait dans le Journal de Lrouville i 895 quand j'ai voulu étudier les conditions auxquelles une fonction $\theta$ de rang $p$ doit satisfaire pour être une fonction $\boldsymbol{\theta}$ spéciale, c'est à dire une fonction $\boldsymbol{\theta}$ de Riemann engendrée par une courbe algébrique de genre $p$.

Enfin c'est sur l'étude des cas de réduction qu'est fondée la démonstration du théorème $B$ qui a été imaginée d'abord par Weicrstrass 
et que nous avons retrouvée, M. Picard et moi, et publiée dans les Comptes-Rendus.

\section{\$ \%. Zéros des fonctions $\theta$.}

Les fonctions intermédiaires peuvent toujours se ramener aux fonctions $\theta$, soit immédiatement, soit après un changement de périodes. J'appelle fonctions $\theta$ les fonctions intermédiaires pour lesquelles $p$ des multiplicateurs se réduisent à des constantes, et les $p$ autres à des exponentielles. La plupart des auteurs ont attribué une importance prépondérante et presque exclusive à celles de ces fonctions où les nombres appelés caractéristiques sont des entiers. J'ai toujours trouvé beaucoup plus commode de m'affranchir de cette restriction et d'attribuer à ces caractéristiques des valeurs quelconques. En revanche j'ai supposé le plus souvent que les $p$ premiers multiplicateurs étaient égaux à $\mathrm{r}$, ce qui ne restreint pas la généralité d'une façon essentielle.

Quoi qu'il en soit, on sait que le nombre des fonctions $\theta$ d'ordre $m$, linéairement indépendantes et ayant mêmes multiplicateurs est égal à $m^{p}$; si on les regarde comme les coordonnées homogènes d'un point dans l'espace à $m^{p}$ - I dimensions, ce point engendrera une variété à $p$ dimensions. J'ai étudié cette variété principalement dans le Journal de Liouville i 895 et j'ai en particulier déterminé son degré de deux manières différentes.

Le nombre des zéros communs à $p$ fonctions $\theta$ d'ordre

est égal à

$$
m_{1}, m_{2}, \ldots, m_{p}
$$

$$
m_{1} m_{2} \ldots m_{p} \cdot p^{\prime}
$$

C'est ce que j'ai démontré dans le tome ro du Bulletin de la Société Mathématique de France.

Dans le Journal de LiouvinLe, j’ai abordé un problème qui contient à la fois comme cas particulier celui dont je viens d'énoncer la solution, et une question résolue autrefois par Riemann.

Soit un système de fonctions abéliennes spéciales au sens du paragraphe précédent. Soient $\boldsymbol{\theta}_{1}\left(v_{i}\right), \boldsymbol{\theta}_{2}\left(v_{i}\right), \ldots, \boldsymbol{\theta}_{\boldsymbol{q}}\left(v_{i}\right) q$ fonctions $\boldsymbol{\theta}$. Soit 
$\boldsymbol{u}_{i}(x)$ l'intégrale abélienne de $\mathrm{I}^{\text {ère }}$ espèce qui sur la courbe de genre $p$ qui engendre ces fonctions $\boldsymbol{\theta}$ correspond à la variable $v_{i}$. Combien les équations

$$
\boldsymbol{\theta}_{k}\left[u_{i}\left(x_{1}\right)+u_{i}\left(x_{2}\right)+\ldots+u_{i}\left(x_{q}\right)\right]=0 \quad(k=1,2, \ldots, q)
$$

où $x_{1}, x_{2}, \ldots, x_{q}$ sont les inconnues, admettent-elles de solutions?

La solution de cette question est donnée par une formule qui contient comme cas particuliers la formule ( $\mathrm{I}$ ) et celle de Riemans.

\section{8. Fonctions spéciales.}

Quellè est la condition nécessaire et suffisante pour qu'une fonction abélienne soit spéciale?

Riemann a démontré que pour les fonctions spéciales, la variété à $p-$ I dimensions:

$$
\theta=0
$$

est une "variété doublement de translations.

Sophus Lie a établi ensuite que cette condition n'est pas seulement nécessaire mais qu'elle est aussi suffisante pour qu'une fonction $\theta$ soit spéciale.

J'ai donné dans le Bulletin de la Société Mathématique de France (I90I) une nouvelle démonstration du théorème de LIE qui me semble plus simple et plus synthétique que celle de ce géomètre.

D'autre part j'ai cherché dans le Journal de Liouvilue i 895 à approfondir cette condition.

J'ai cherché à l'exprimer en fonction des périodes, et dans les cas voisins $\mathrm{du}$ cas singulier elliptique je suis parvenu à former les premiers termes du développement de la fonction des périodes qui, égalée à zéro, exprime que cette condition est remplie.

J'ai d'ailleurs étudié la courbe qui engendre cette variété de translation; cette courbe satisfait à $p-\mathrm{I}$ équations de la forme:

$$
\boldsymbol{\theta}\left(v_{i}-e_{i k}\right)=0
$$

$(k=1,2, \ldots, p-1)$

les $e$ étant des constantes. Mais ces équations ne suffisent pas pour déterminer cette courbe; elles définissent une courbe décomposable dont la 
courbe envisagée n'est qu'une composante. Tous ces points étant presque évidents, j’ai cherché à me rendre compte des circonstances de cette décomposition.

\section{Somme des zéros.}

Dans le tome 8 de l'American Journal, j'ai démontré, en m'appuyant sur une généralisation du théorème d'ABEL, que la somme des zéros communs à $p$ fonctions intermédiaires est une constante, et ne dépend que des multiplicateurs de ces fonctions.

J'ai ensuite, en remarquant qu'on est toujours infiniment près d'un cas de réduction, déterminé la valeur de cette constante.

Je voudrais déduire de là une conséquence.

Soient

$$
\theta, \theta_{1}, \theta_{2}, \ldots, \theta_{p}
$$

$p+1$ fonctions $\theta$ ayant mêmes multiplicateurs.

Considérons maintenant les zéros communs aux $p$ dernières fonctions; ces zéros seront donnés par les équations

$$
\boldsymbol{\theta}_{1}\left(v_{i}\right)=\boldsymbol{\theta}_{2}\left(v_{i}\right)=\ldots=\boldsymbol{\theta}_{p}\left(v_{i}\right)=\mathrm{o} .
$$

Soit

$$
v_{i}=g_{i k}
$$

une de ces solutions; l'indice $i$ varie de I à $p$, l'indice $k$ de 1 à $N$,

$$
N=m^{p} \mid \underline{p}
$$

étant le nombre des solutions des équations (I) et $m$ l'ordre des fonctions $\boldsymbol{\theta}$ considérées.

Formons maintenant les équations suivantes:

$$
\text { (2) } \boldsymbol{\theta}_{1}\left(v_{i}\right)+\varepsilon_{1} \boldsymbol{\theta}\left(v_{i}\right)=\boldsymbol{\theta}_{2}\left(v_{i}\right)+\varepsilon_{2} \boldsymbol{\theta}\left(v_{i}\right)=\ldots=\boldsymbol{\theta}_{p}\left(v_{i}\right)+\varepsilon_{p} \boldsymbol{\theta}\left(v_{i}\right)=0
$$

les $\varepsilon$ étant des constantes. Ces équations auront $N$ solutions; soit

$$
v_{i}=g_{i k}^{\prime}
$$

l'une de ces solutions. Les fonctions $\boldsymbol{\theta}_{k}+\boldsymbol{\varepsilon}_{k} \boldsymbol{\theta}$ ont mêmes multiplicateurs que les fonctions $\boldsymbol{\theta}_{k}$. On aura donc

$$
\Sigma g_{i k}=\Sigma g_{i k}^{\prime} \text {. }
$$


Si les $\varepsilon$ sont très petits, nous pourrons poser

$$
g_{i k}^{\prime}-g_{i k}=\delta g_{i k}
$$

et les $\delta g_{i k}$ seront très petits de l'ordre des $\varepsilon$; on aura donc en négligeant les quantités de l'ordre de $\varepsilon^{2}$ :

$$
\begin{aligned}
\theta_{1}\left(g_{i k}^{\prime}\right) & =\theta_{1}\left(g_{i k}\right)+\sum \delta g_{j k} \frac{d \theta_{1}}{d v_{j}}=\sum \delta g_{j k} \frac{d \theta_{1}}{d v_{j}}, \\
\varepsilon_{1} \theta\left(g_{i k}^{\prime}\right) & =\varepsilon_{1} \theta\left(g_{i k}\right) .
\end{aligned}
$$

Dans les dérivées $\frac{d \theta_{1}}{d v_{j}}$, les $v_{i}$ doivent être remplacés par $g_{i k}$.

Les équations (2) peuvent donc s'écrire:

$$
\sum \delta g_{j k} \frac{d \theta_{q}}{d v_{j}}+\varepsilon_{q} \theta\left(g_{i k}\right)=0
$$

Soit $\Delta\left(v_{i}\right)$ le déterminant $\operatorname{des} \frac{d \theta_{q}}{d v_{i}}$, c'est à dire le déterminant fonctionnel des $\boldsymbol{\theta}_{q}$ par rapport aux $v$. Soit $\Delta_{q j}\left(v_{i}\right)$ le mineur de ce déterminant correspondant à l'élément $\frac{d \theta_{q}}{d v_{j}}$; les équations (4) nous donnent:

$$
-\frac{\theta\left(g_{i k}\right)}{\Delta\left(g_{i k}\right)} \Sigma \varepsilon_{q} \Delta_{q j}\left(g_{i k}\right)
$$

En vertu de l'équation (3) on a $\Sigma \delta g_{i k}=0$; on aura donc aussi quels que soient les indices $q$ et $j$ :

$$
\sum \boldsymbol{\theta}\left(g_{i k}\right) \frac{\Delta_{g j}\left(g_{i k}\right)}{\Delta\left(g_{i k}\right)}=0
$$

La sommation doit être étendue aux $N$ solutions des équations (I).

Pour nous rendre compte de la signification de ce théorème, voyons ce qu'il devient dans le cas des fonctions elliptiques $(p=1)$; on a alors:

$$
\Delta_{g j}=\mathrm{I}, \quad \Delta\left(v_{1}\right)=\theta_{1}^{\prime}\left(v_{1}\right)
$$

et notre équation devient:

$$
\sum \frac{\theta\left(g_{1 k}\right)}{\theta_{1}^{\prime}\left(g_{1 k}\right)}=0 .
$$

La fonction

$$
\frac{\theta}{\theta_{1}}
$$


est alors une fonction doublement périodique admettant pour pôles les points $g_{1 k}$; le résidu correspondant est précisément

$$
\frac{\theta\left(g_{1 k}\right)}{\theta_{1}^{\prime}\left(g_{1 k}\right)}
$$

L'équation (6) exprime donc le théorème bien connu d'après lequel la somme des résidus d'une fonction doublement périodique est nulle.

A ce point de vue, l'équation (5) peut être regardée comme la généralisation du théorème des résidus.

Nous avons supposé jusqu'ici que les $p+\mathrm{I}$ fonctions $\boldsymbol{\theta}, \boldsymbol{\theta}_{1}, \boldsymbol{\theta}_{\mathbf{2}}, \ldots, \boldsymbol{\theta}_{p}$ avaient mêmes multiplicateurs. Supposons maintenant que $\theta$ et $\theta_{1}$ aient mêmes multiplicateurs; mais ne supposons plus que les autres fonctions aient mêmes multiplicateurs, ni même qu'elles soient de même ordre.

Reprenons les équations ( $\mathrm{I}$ ) et (2) mais en faisant

$$
\varepsilon_{2}=\varepsilon_{3}=\ldots=\varepsilon_{p}=0 \text {. }
$$

Les fonctions

$$
\theta_{1}+\varepsilon_{1} \theta, \theta_{2}, \ldots, \theta_{p}
$$

ayant mêmes multiplicateurs que

$$
\theta_{1}, \theta_{2}, \ldots, \theta_{p}
$$

les équations (3) subsistent, et par conséquent aussi l'équation (5), mais pour $q=\mathrm{I}$ seulement.

Soit

$$
F=\frac{\theta}{\theta_{1}}
$$

Regardons $F$ comme une fonction de $v_{j}$, les autres $v$ étant supposés exprimés en fonctions de $v_{j}$ par les équations

$$
\theta_{2}=\theta_{\mathrm{s}}=\ldots=\theta_{p}=0 \text {. }
$$

Notre fonction $F$ admettra comme infinis

$$
v_{j}=g_{j k} \text {. }
$$

Quel sera le résidu correspondant? C'est la limite de:

$$
H=\left(v_{j}-g_{j k}\right) \boldsymbol{F}
$$

quand $v_{j}$ tend vers $g_{j k}$, de façon que $\boldsymbol{\theta}_{2}, \boldsymbol{\theta}_{3}, \ldots, \boldsymbol{\theta}_{p}$ restent nuls.

Acta mathomatica. 26. Imprimé le 10 juin 1902. 
Soit

$$
\grave{\partial} v_{i}=v_{i}-g_{i k}
$$

nous aurons

$$
\begin{gathered}
\sum \partial v_{i} \frac{d \theta_{q}}{d v_{i}}=0 \\
\theta_{1}=\sum \partial v_{i} \frac{d \theta_{1}}{d v_{i}}=\frac{H}{\theta} \partial v_{j} .
\end{gathered}
$$

De cette équation nous tirons:

$$
\Delta^{\prime}=\mathrm{o}
$$

$\Delta^{\prime}$ étant le déterminant fonctionnel $\Delta$ où $\frac{d \theta_{1}}{d v_{j}}$ est remplacé par $\frac{d \theta_{1}}{d v_{j}}-\frac{H}{\theta}$. De là nous tirons:

$$
H=\boldsymbol{\theta}\left(v_{i}\right) \frac{\Delta_{1 j}\left(v_{i}\right)}{\Delta\left(v_{i}\right)}
$$

et à la limite pour le résidu:

$$
\boldsymbol{\theta}\left(g_{i k}\right) \frac{\Delta_{1 j}\left(g_{i h}\right)}{\Delta\left(g_{i k}\right)}
$$

L'équation (5) exprime donc bien que la somme des résidus (entendus au sens que nous venons de préciser) est nulle. 